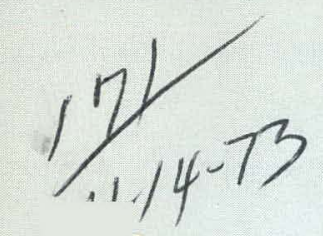

th. 418

K-1847

\title{
PERFORMANCE OF COMPACT HEAT TRANSFER SURFACES - FINNED AND BARE TUBE BANKS
}

B. Cox

UNION CARBIDE
OAK RIDGE GASEOUS DIFFUSION PLANT OAK RIDGE. TENNESSEE

\section{MASTER}




\section{DISCLAIMER}

This report was prepared as an account of work sponsored by an agency of the United States Government. Neither the United States Government nor any agency Thereof, nor any of their employees, makes any warranty, express or implied, or assumes any legal liability or responsibility for the accuracy, completeness, or usefulness of any information, apparatus, product, or process disclosed, or represents that its use would not infringe privately owned rights. Reference herein to any specific commercial product, process, or service by trade name, trademark, manufacturer, or otherwise does not necessarily constitute or imply its endorsement, recommendation, or favoring by the United States Government or any agency thereof. The views and opinions of authors expressed herein do not necessarily state or reflect those of the United States Government or any agency thereof. 


\section{DISCLAIMER}

Portions of this document may be illegible in electronic image products. Images are produced from the best available original document. 
Printed in the United States of America. Available from National Technical Information Service

U.S. Department of Commerce

5285 Port Royal Road, Springfield, Virginia 22151

Price: Printed Copy $\$ 5.45$; Microfiche $\$ 1.45$

This report was prepared as an account of work sponsored by the United States Government. Neither the United States nor the United States Atomic Energy Commission, nor any of their employees, nor any of their contractors, subcontractors, or their employees, makes any warranty, express or implied, or assumes any legal liability or responsibility for the accuracy, completeness or usefulness of any information, apparatus, product or process disclosed, or represents that its use would not infringe privately owned rights. 


\section{PERFORMANCE OF COMPACT HEAT TRANSFER SURFACES-} FINNED AND BARE TUBE BANKS

B. $\operatorname{Cox}^{*}$

Thermal Systems Development Group Gaseous Diffusion Development Division

Oak Ridge Gaseous Diffusion Plant Union Carbide Corporation

Oak Ridge, Tennessee

Prepared for the U. S. Atomic Energy Commission under U. S. Government Contrast W-7405 eng 26

\footnotetext{
*Present.] y lorated in the Test Unit Assembly Department, Separation Systems Division.
} 
THIS PAGE

\section{WAS INTENTIONALLY LEFT BLANK}


ABSTRACT

Heat transfer and flow friction characteristics were determined experimentally for seven high-finned tube banks. A variety of fin types were represented: plate fins, integral smooth helical fins, integral serrated fins, and helical serrated fins. The experimental data results plus data from the open literature are used to determine the effects that tube pitch has on tube bank performance. 
THIS PAGE

\section{WAS INTENTIONALLY LEFT BLANK}


CONTENTS

$\underline{\text { Page }}$

LIST OF FIGURES . . . . . . . . . . . . . . . . . . . . 7

NOMENCLATURE . . . . . . . . . . . . . . . . . . . . 9

INTRODUCTION AND SUMMARY . . . . . . . . . . . . . . . II

BASIC DESIGN DATA . . . . . . . . . . . . . . . . . II

ANALYSIS OF RESULTS . . . . . . . . . . . . . . . . . . . . . . 19

Performance Equations . . . . . . . . . . . . . . . 19

Effects of Tube Pitch . . . . . . . . . . . . . . . . . 21

Comments . . . . . . . . . . . . . . . . . . . . 27

CONCLUSIONS . . . . . . . . . . . . . . . . . . 28

APPENDIX A. GEOMETRIC AND EXPERIMENTAL DAIA . . . . . . • . • 29

APPENDIX B. LITERATURE IUBE BANK RESULTS . . . . . . . . . . . . 39

ACKNOWLEDGMENTS . . . . . . . . . . . . . . . . . . 44

REFERENCES ........................ . . 45 
THIS PAGE

\section{WAS INTENTIONALLY LEFT BLANK}


LIST OF FIGURES

Figure

Title

Page

1

Surface 6, Integral Tube-Helical Fin

2

Surface 8, Expanded-Tube-Continuous Fin

Surface 9, Expanded Tube-Louvered Fin

Surface 10, Integral Flat Tube-Serrated Fin

Surface 1l, Welded Tube-Serrated Fin

Șurface 12, Welded Tube-Serrated Fin

8 Heat Transfer Rates for Tube Banks AB-1, 2, 3, 4, 5, 6. Designations from table B-I.

9 Heat Transfer Rates for Tube Banks J-A, B, C, D, E. Designations from table B-l.

10 Heat Transfer Rates for Tube Banks CoX-1, 2, 3.

Designations for Surfaces 4, 5, 13 in table 1 .

Heat Transfer Rates for Tube Banks $\mathrm{KL}-5,6,7,8$, $9,10,13,14$. Designations from table B-2. 
THIS PAGE

\section{WAS INTENTIONALLY}

LEFT BLANK 
NOMENCLATURE

Basic Symbols

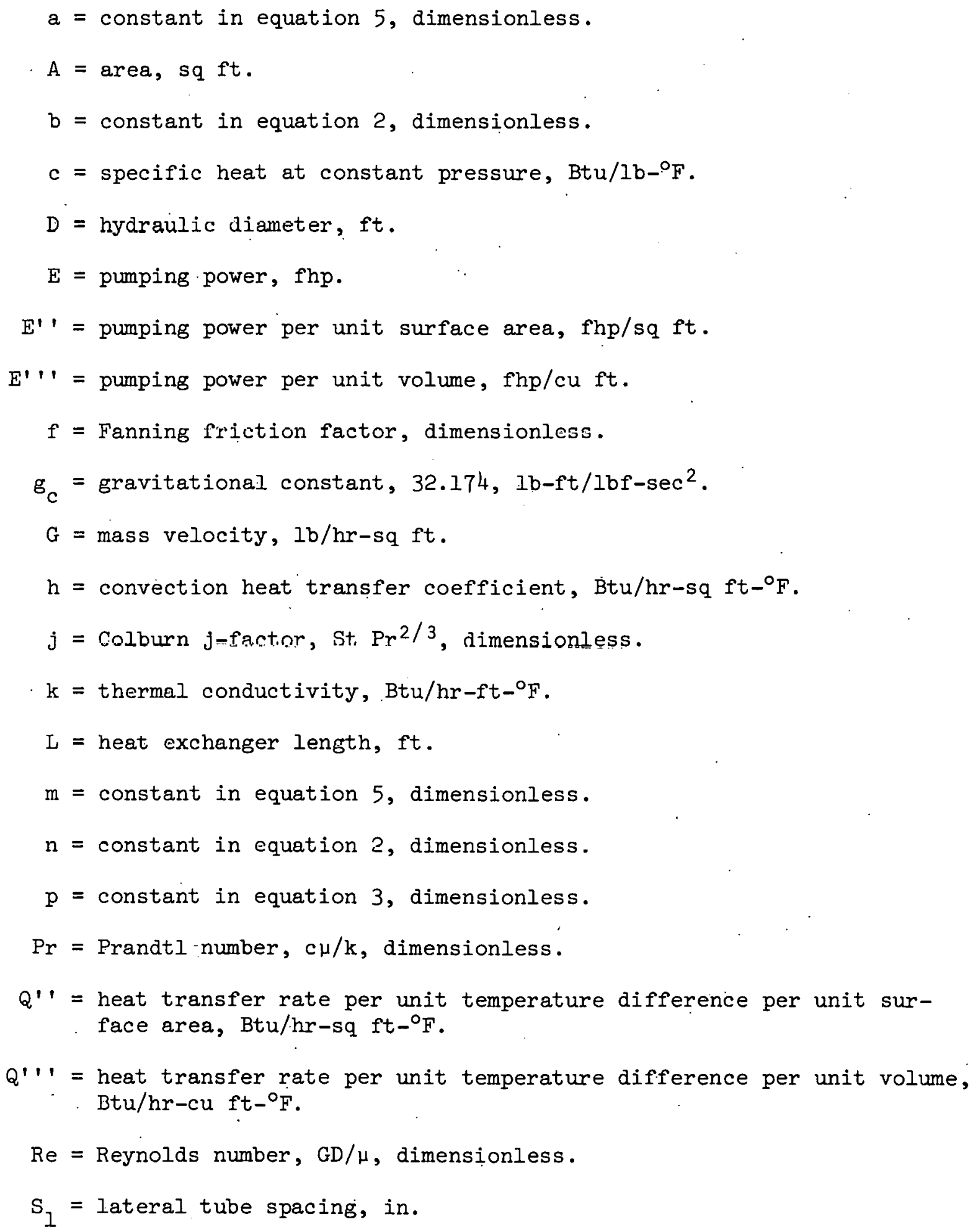




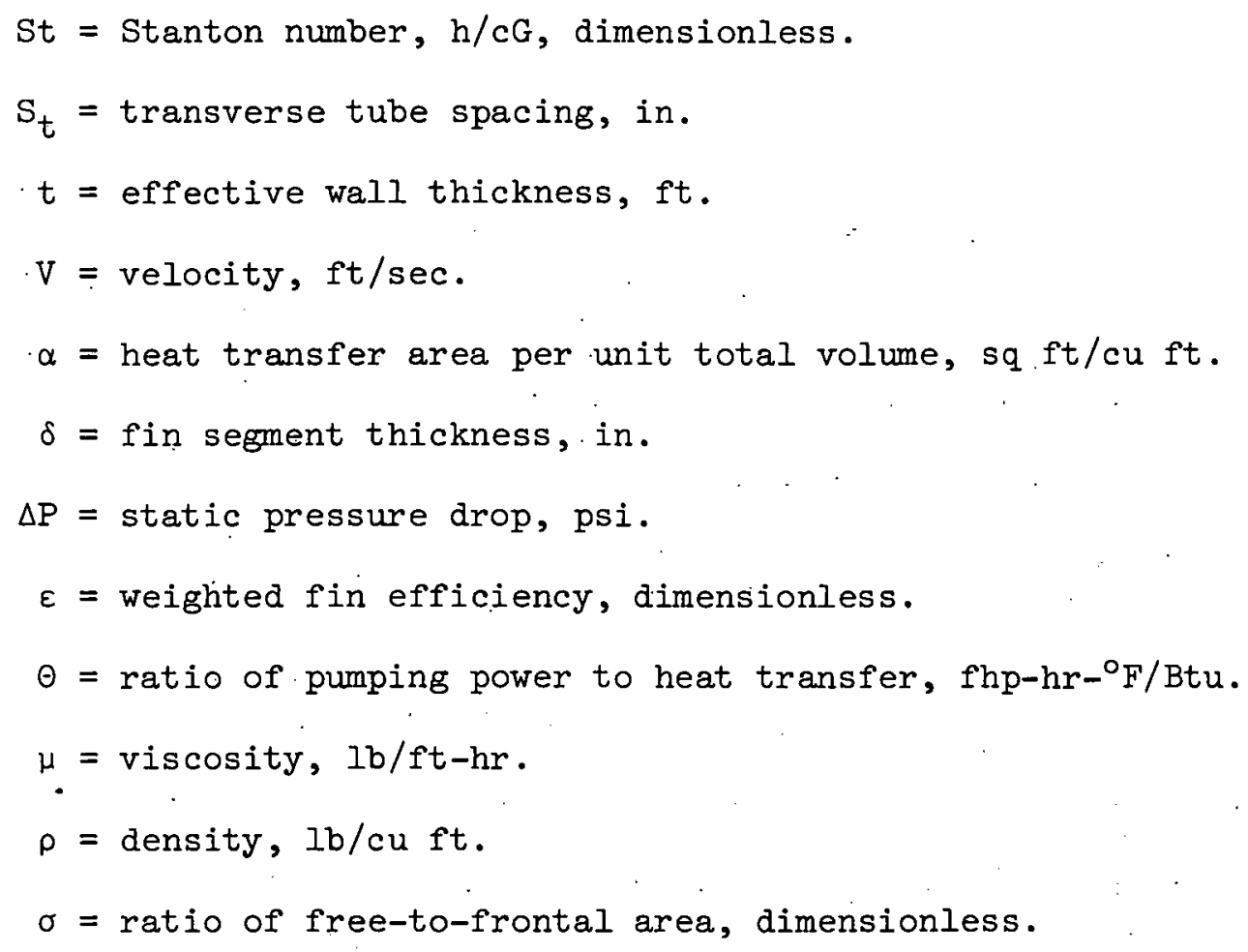

Subscripts

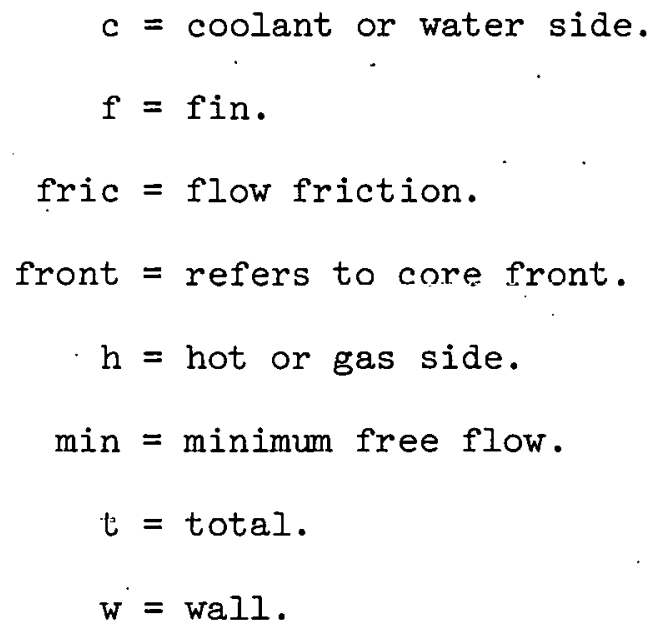




\author{
PERFORMANCE OF COMPACT HEAT TRANSFER SURFACES- \\ FINNED AND BARE TUBE BANKS
}

\title{
INTRODUCTION AND SUMMARY
}

The increasing need for compact heat exchangers has resulted in greater efforts by industry to manufacture and evaluate new types of heat transfer surfaces. For example, a significant part of the expansion program for the nation's gaseous diffusion plants involves the design of new and more efficient heat exchangers. Because of their high surface area densities and subsequent low volume requirements, high-finned tube surfaces appeared attractive for these designs. High-finned tubes also find applications in economizers, air-conditioners, gas-cooled nuclear reactors, air-cooled condensers, plus others discussed in reference 1.

This study documents the latest results from the Oak Ridge Gaseous Diffusion Plant (ORGDP) program to evaluate compact heat transfer surfaces. Previous reports ${ }^{2}, 3$ described the experimental apparatus and the data reduction procedure. Those studies emphasized performance comparisons of different types of compact surfaces, including finned tubes: The present work emphasizes performance comparisons of different arrangements of finned tubes.

\section{BASIC DESIGN DATA}

Experimental heat transfer and pressure drop data were taken with air flowing across seven different banks of finned tubes. The data were reduced to the dimensionless form of Colburn j-factors and Fanning friction. factors. The basic design data are shown graphically in figures 1-7. Descriptions of the geometries accompany the graphs. The experimental data and results are tabulated in Appendix A.

Table 1 describes the 13 surfaces tested during the present program. It should be noted that several of the surfaces were retested but retained their original designation number. Thus far, the compact surfaces tested have been numbered consecutively. (If many more are tested this practice could become cumbersome and a labeling system similar to that of reference 4 might be preferable.)

Several other sets of finned tube data were taken from the open literature and transformed to $f$ and $j$ versus Reynolds number curves. One set was from the work of Ackerman and Brunsvold, ${ }^{5}$ who reported results with studded tubes. Another set was from the work of Jameson, 6 . who reported results with helically finned tubes. For bare tube comparisons, the $f$ and $f$ curves of Kays and London ${ }^{4}$. were used. Appendix B discusses the derivations of these $f$ and $j$ curves. 

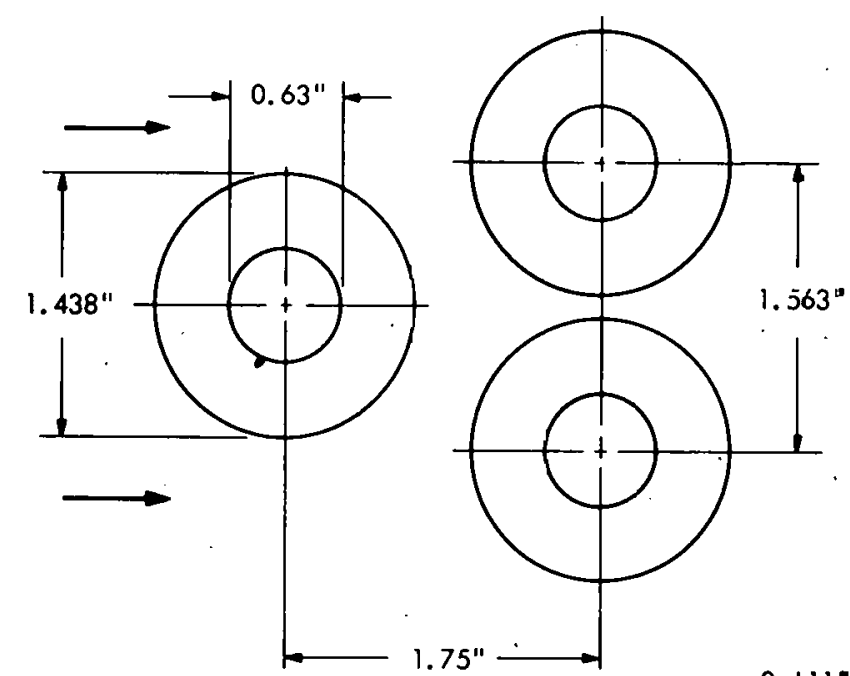

$0.111 *$
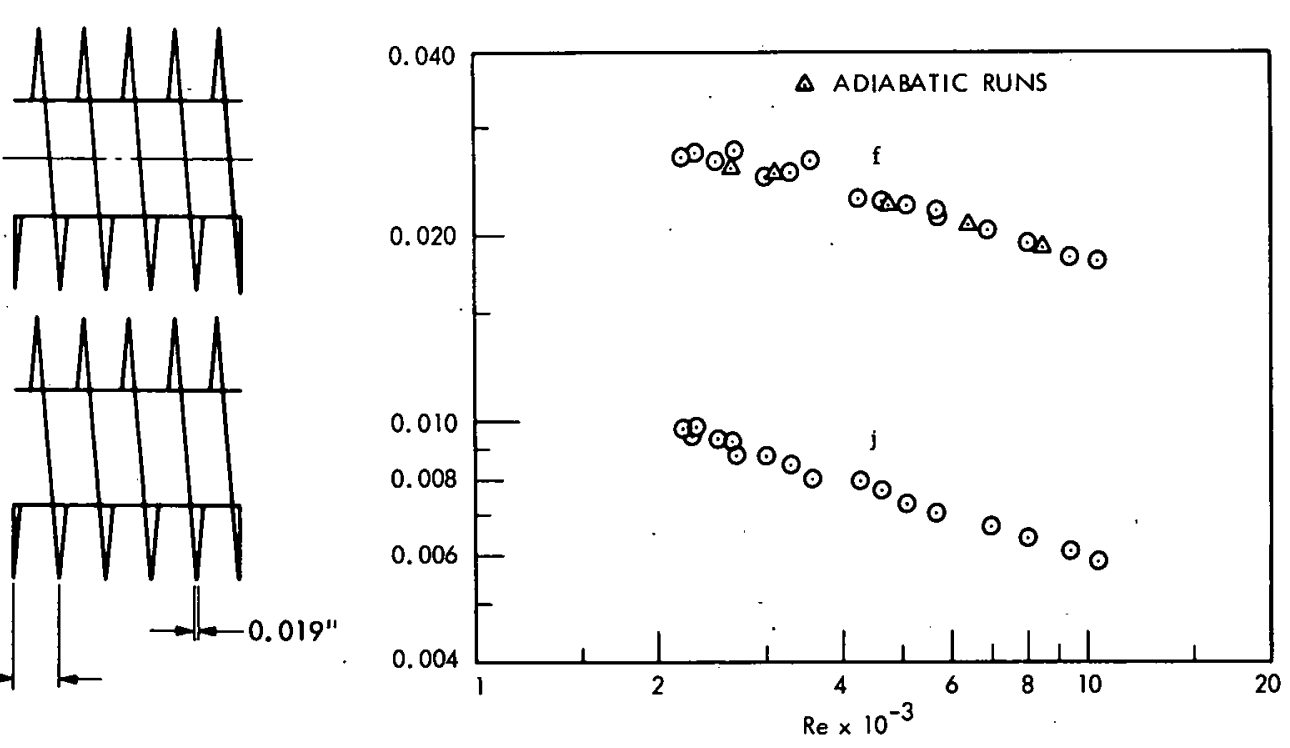

TUBE O.D. $=0.63 \mathrm{IN}$.

FIN PITCH $=9.0$ PER IN

$\delta \quad=0.0191 \mathrm{~N}$

$A_{f} / A_{t}=0.937$

D $\quad=0.01780 \mathrm{FT}$

$\sigma \cdot=0.5086$

$=114.3 \mathrm{FT}^{2} / \mathrm{FT}^{3}$

Figure 1

SURFACE 6, INTEGRAL TUBE - HELICAL FIN 
DWG. NO. G-73-18
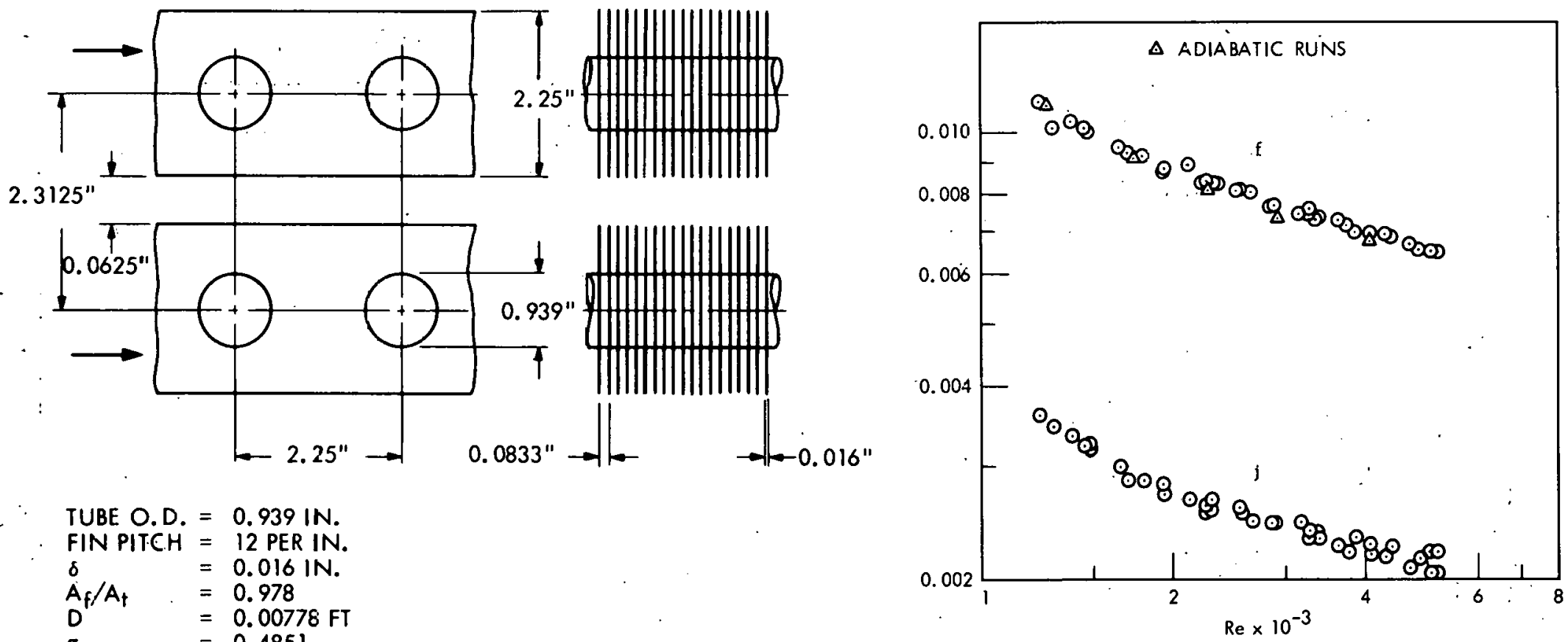

Figure 2

SIJRFACE 8, EXPANDED TUBE - CONTINUOUS FIN 

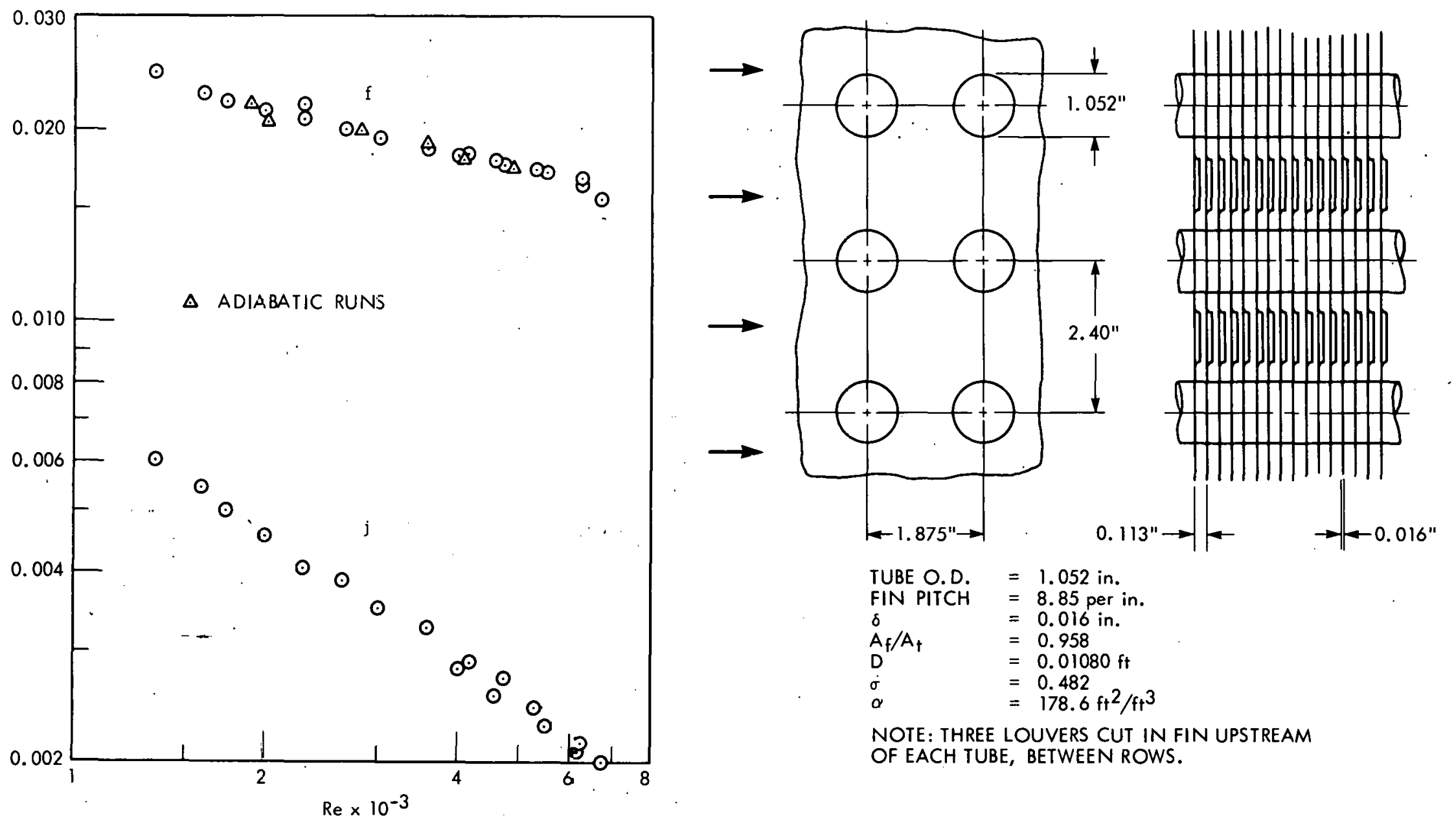

Figure 3

SURFACE 9, EXPANDED TUBE - LOUVERED FIN 
DWG. NO. G-73-19

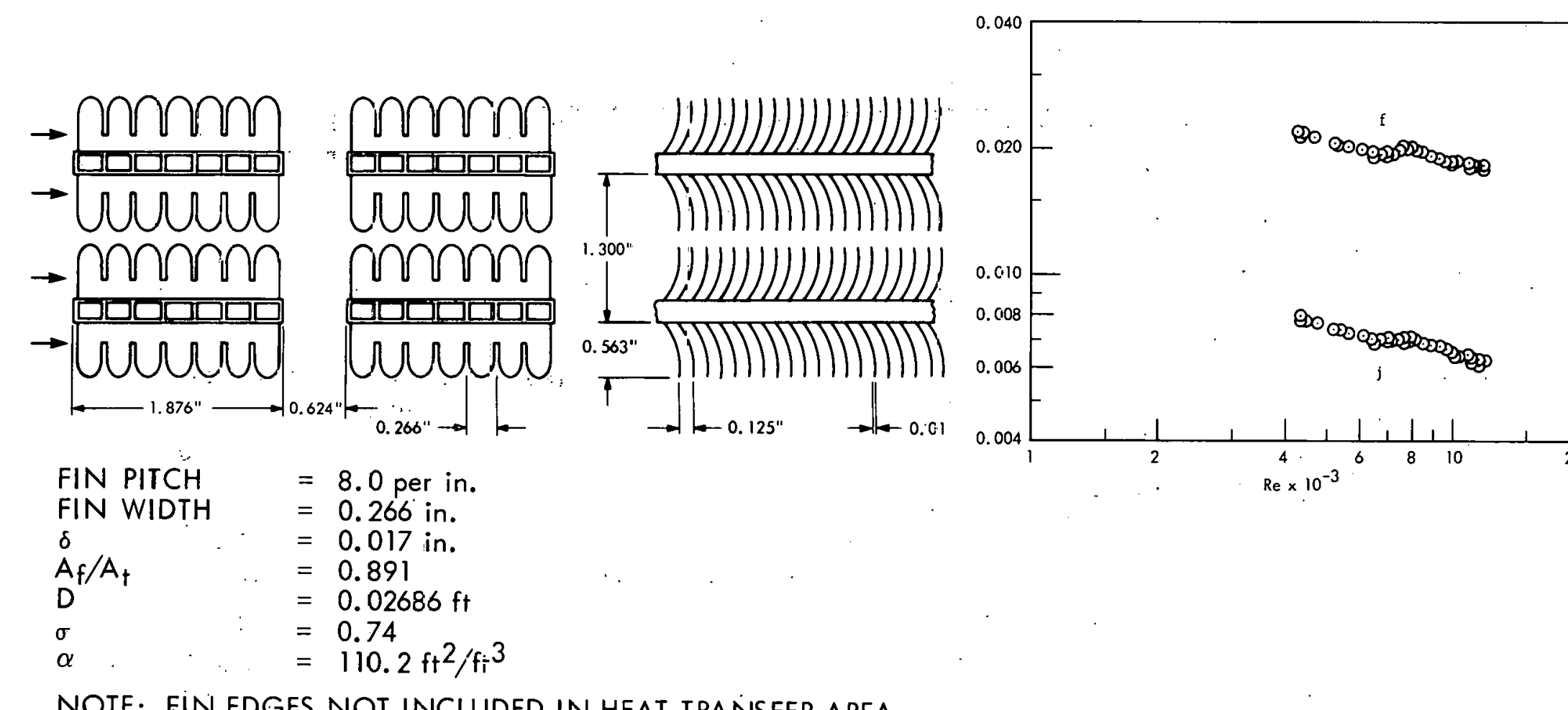

Figure 4

SURFACE 10, INTEGRAL FLAT TUBE - SERRATED FIN 
DWG. NO. G-73-22
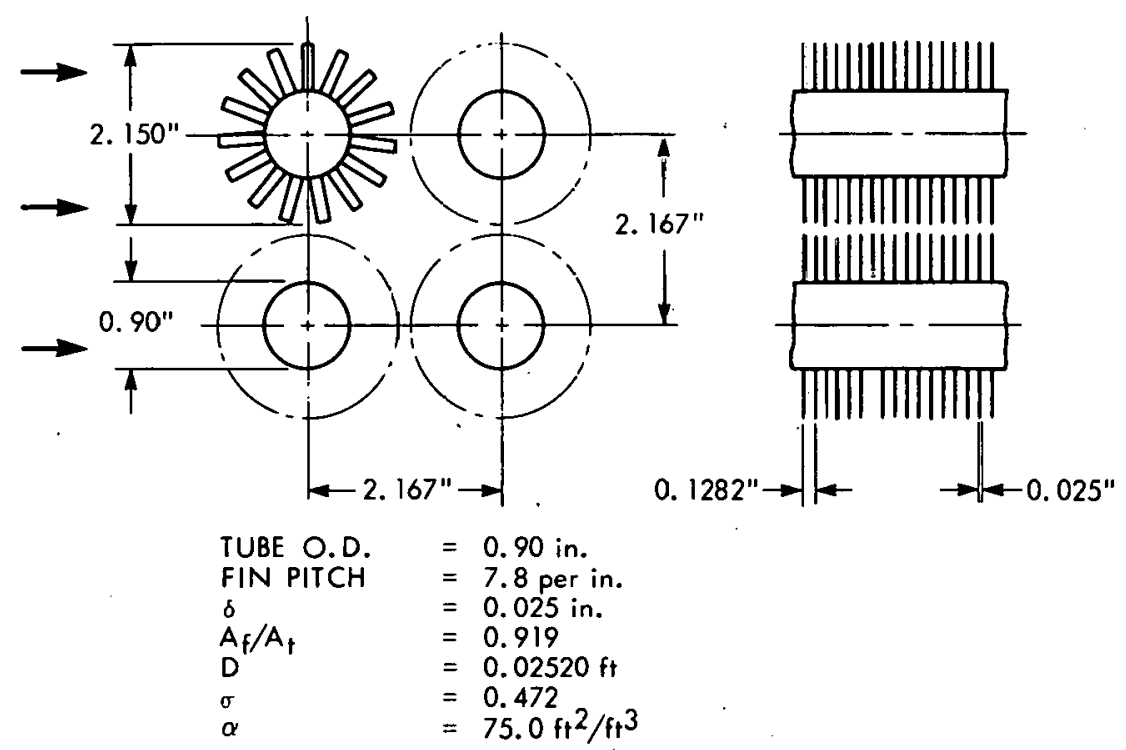

NOTE: POOR BOND BETWEEN TUBE AND FINS.

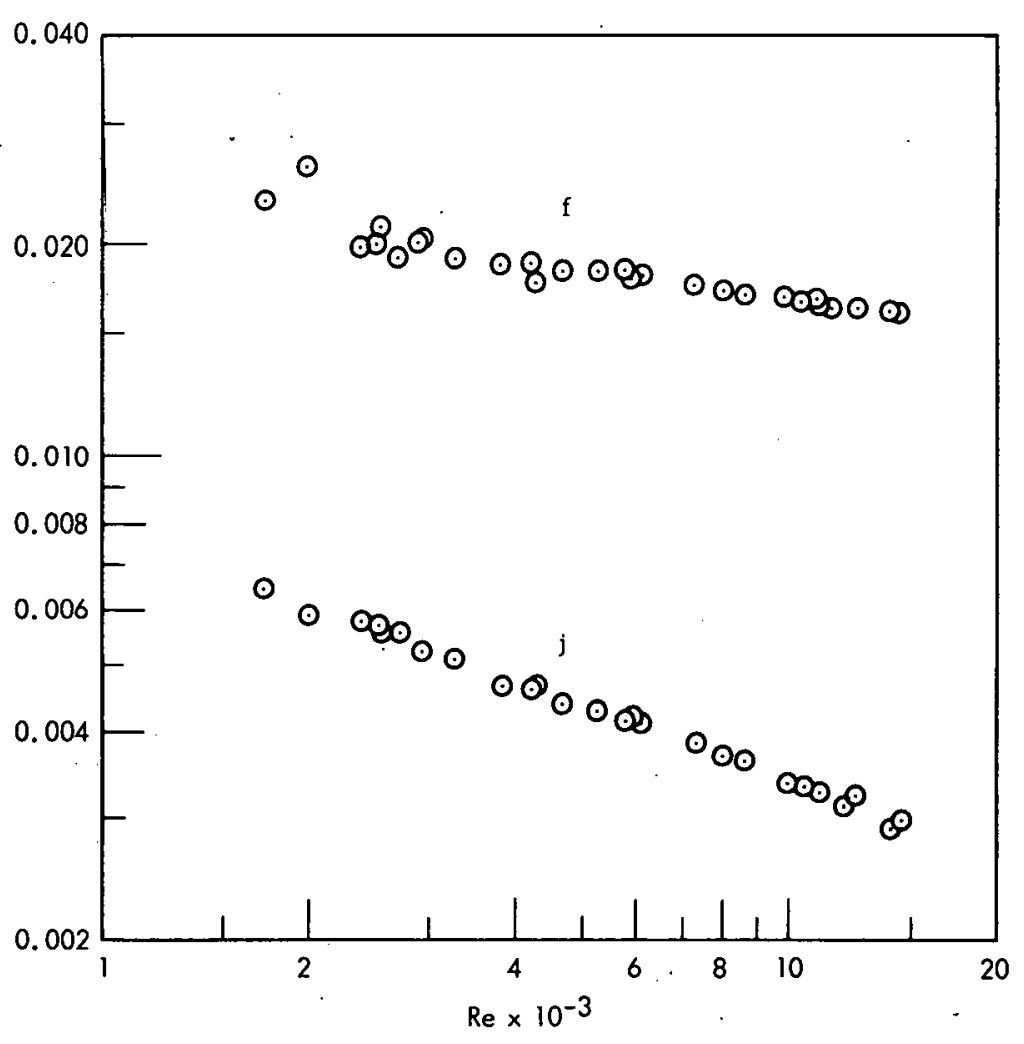

Figure 5

SURFACE 11 , WELDED TUBE - SERRATED FIN 
DWG. NO. G-73-21
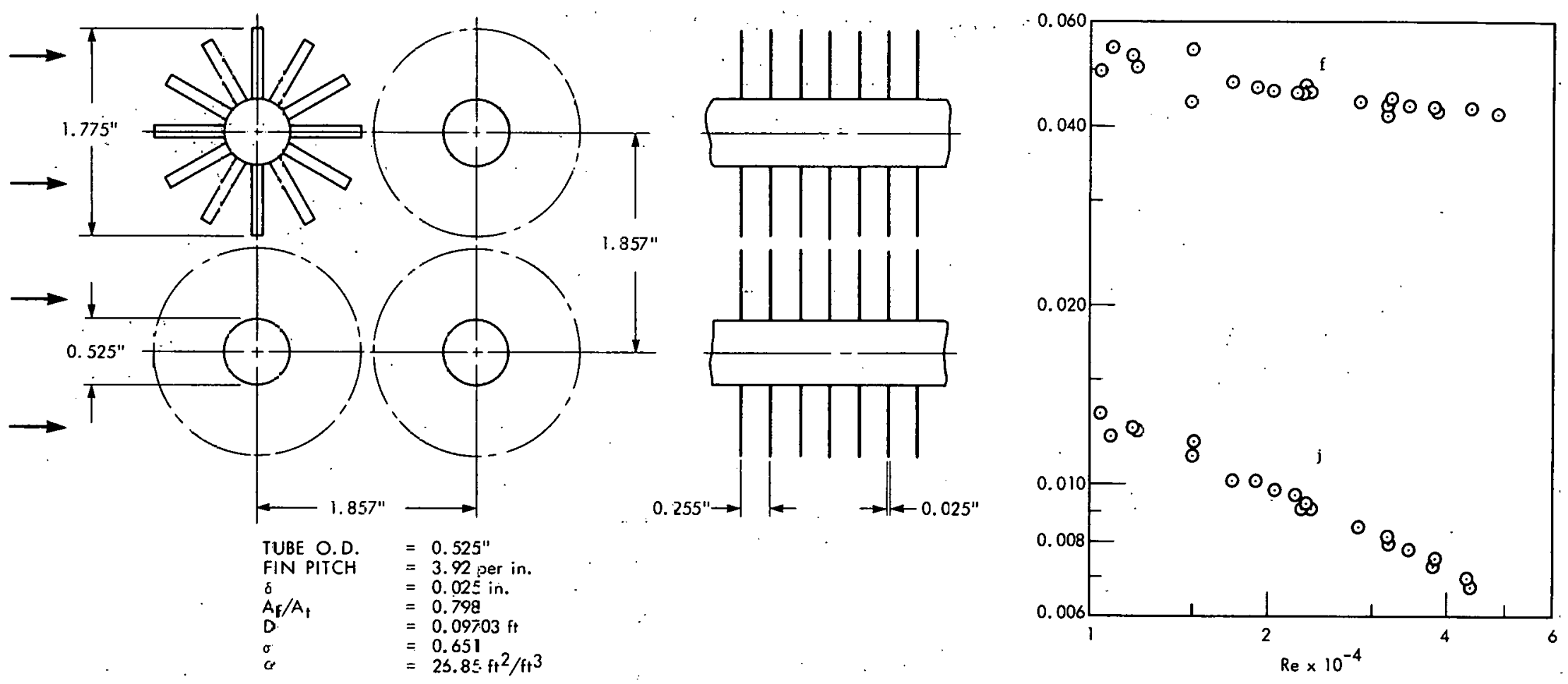

Figure 6

SURFACE 12, WELDED TUBE - SERRATED FIN 
DWG. NO. G-73-20

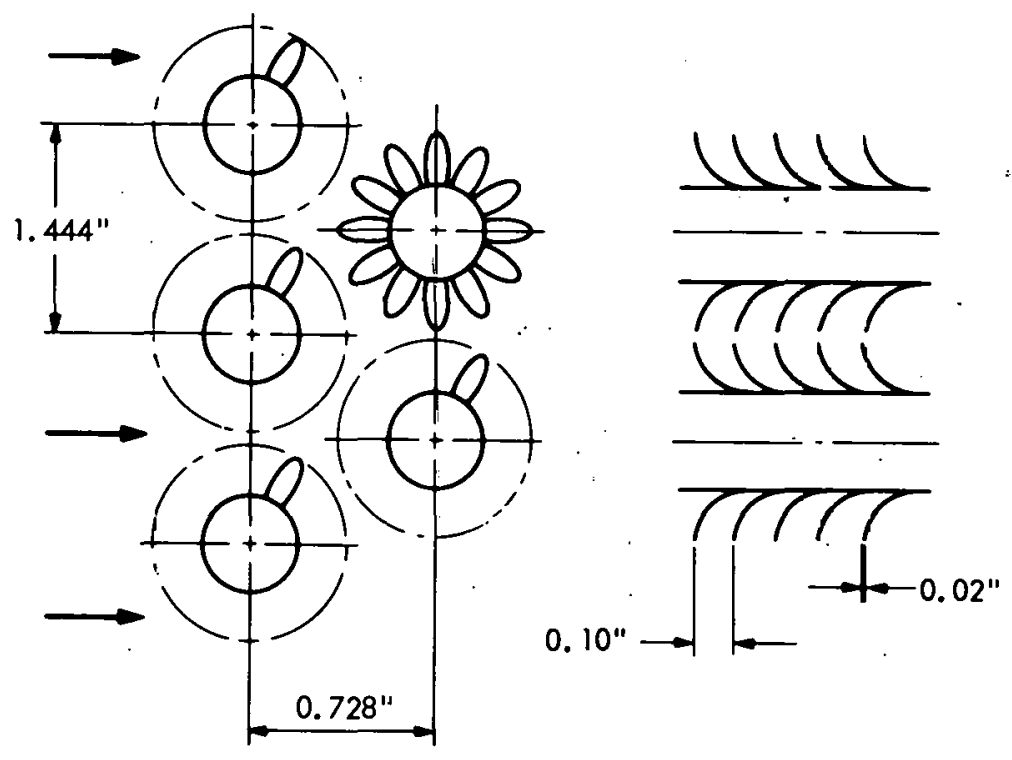

$$
\begin{array}{ll}
\text { TUBE O.D. } & =0.505 \mathrm{in} . \\
\text { FIN PITCH } & =10.0 \mathrm{per} \text { in. } \\
\text { FIN WIDTH } & =0.115 \mathrm{in} . \\
\delta & =0.02 \mathrm{in.} \\
A_{f} / A_{t} & =0.834 \\
D & =0.02587 \mathrm{ft} \\
\sigma & =0.581 \\
\alpha & =89.84 \mathrm{ft}^{2} / \mathrm{ft}^{3}
\end{array}
$$

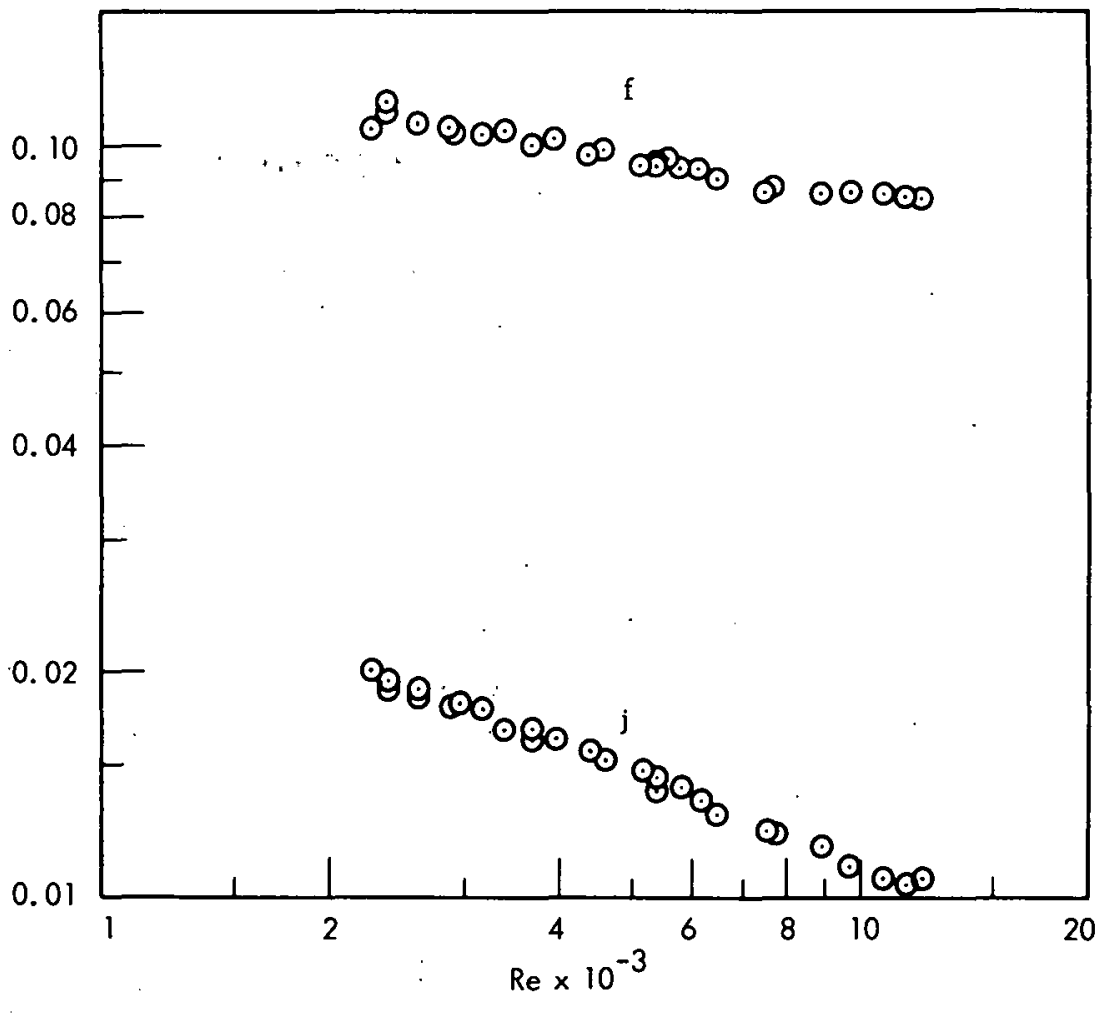

NOTES: FIN EDGES NOT INCLUDED IN HEAT TRANSFER AREA. FLOW AREA IN TRANSVERSE SPACES EQUALS FLOW AREA IN DIAGONAL SPACES.

Figure 7

SURFACE 13, INTEGRAL TUBE - SERRATED FIN 
Table 1

SUMMARY OF EXPERIMENTAL HEAT TRANSFER SURFACES

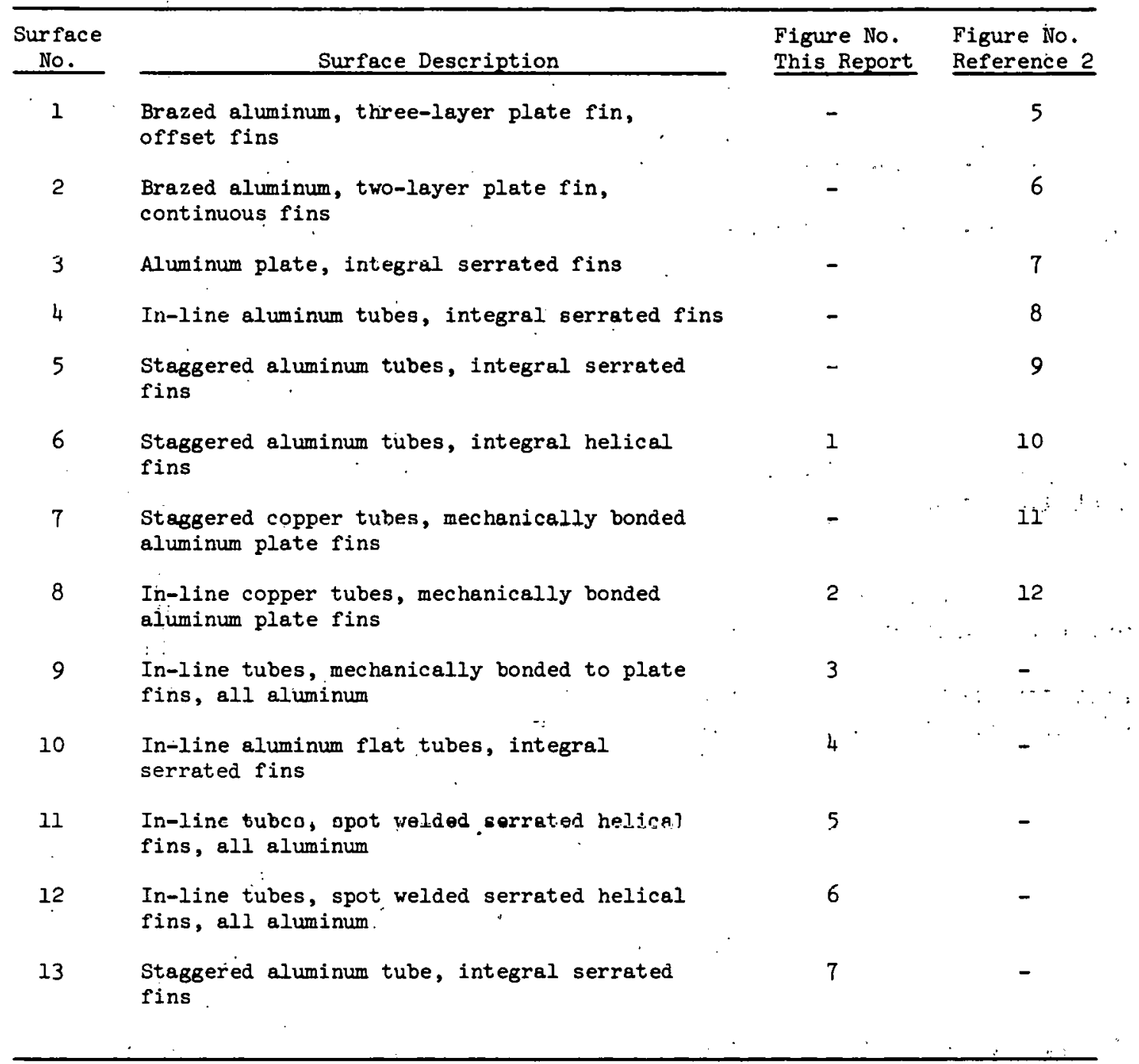

\section{ANALYSIS OF RESULTS}

\section{PERFORMANCE EQUATIONS}

The methods used in determining tube bank performance were those described in reference 3. Pertinent equations from that reference are as follows:

Colburn $j$ factors were defined by

$$
j=\frac{h}{c G} \operatorname{Pr}^{2 / 3} \text {. }
$$


If experimental $\mathrm{j}$ factors are plotted versus Reynolds number, it is found that the data can be approximated by equations of the form

$$
j=b \operatorname{Re}^{n},
$$

or

$$
S t=b e^{n-} \operatorname{Pr}^{p}
$$

where $b$ and $n$ are constants and $p=-2 / 3$ for this study.

Fanning friction factors were defined by the expression

$$
f=\frac{\Delta P_{\text {fric }}}{4 \frac{L}{D} \frac{G^{2}}{2 \rho g_{c}}} .
$$

If experimental $f$ factors are plotted versus Reynolds number, it is found that the data can again be approximated by equations of the form

$$
f=a R e^{m} .
$$

where a and $m$ are constants.

Heat transfer rates per unit temperature difference were based on a unit of surface area and on a unit of volume:

$$
\begin{aligned}
Q^{\prime} & =\frac{\varepsilon k b R e^{n+1} P r^{p+1}}{D}, \\
Q^{\prime}{ }^{\prime} & =\frac{4 \sigma \varepsilon k b R e^{n+1} P r^{p+1}}{D^{2}} .
\end{aligned}
$$

Pumping power required to overcome fluid flow friction was calculated using the following expressions:

$$
\begin{aligned}
E & =\Delta P_{\text {fric }}{ }^{A} \min \\
E^{\prime \prime} & =\frac{\mu^{3} a R e^{m+3}}{2 D^{3} \rho^{2} g_{c}} \cdots \\
E^{\prime \prime} & =\frac{2 \sigma \mu^{3} a R e^{m+3}}{D^{4} \rho^{2} g_{c}}
\end{aligned}
$$

Equations (8), (9), and (10) represent total pumping power, pumping power per unit of surface area, and pumping power per unit of volume, respectively. 
The ratio of energy expended to energy transferred is calculated by

$$
\theta=\frac{E^{\prime \prime}}{Q^{\prime \prime}}=\frac{E^{\prime \prime \prime}}{Q^{\prime \prime \prime}}=\frac{\mu^{3} a R e^{m-n+2}}{2 D^{2} \rho^{2} g_{C} \varepsilon k b P^{p+1}} .
$$

\section{EFFECTS OF TUBE PITCH}

To evaluate the effects that tube pitch had on tube bank performance, the method described in reference 3 was used: values of $Q^{\prime \prime}$ and $Q^{\prime \prime}$ ' were plotted versus $\theta$, equations (6), (7), and (11). This type of graph gives. a direct comparison of the heat transfer area and volume required by different tube banks at a given power expenditure. For a given mass throughput, values of face mass velocity then indicate the relative frontal areas required for the tube bank heat exchanger. Thermophysical properties were evaluated at standard conditions.*

On the above basis, Ackerman and Brunsvold's data are shown in figure 8; Jameson's in figure 9; Cox and Jallouk's in figure 10; and Kays and London's in figure 1i. Tube arrangement designations are from table B-l.

With the exception of in-line $A B-4$, the figure 8 heat transfer rates per unit area, $Q^{\prime \prime}$, are very close to one another. The differences may well be within the experimental accuracy of the data, but some trends are apparent:

i. If $S_{t}$ is held constant, a decrease in $S_{1}(A B-1,3,5)$, requires either an increased power expenditure or an increașed frontal area at a given Q''.

2. If $S_{1}$ is held constant, a decrease in $S_{t}(A B-1,6 ; A B-2,5)$ increases $Q^{\prime}$ ' but also increases the pumping power for a given frontal area.

3. The staggered and in-line arrangements, which have equal $S_{t}, S_{l}$, and $\alpha(A B-3,4)$, show the staggered arrangement to have both a higher $Q^{\prime} '$ and higher pumping power requirement at the same Geace.

4. The highest performance surfaces $(A B-3,6)$ are closest to an equilateral triangle arrangement. The closer packed AB-6 requires either a larger frontal area or higher pumping power expenditure at comparable values of $Q^{\prime \prime}$.

5. Volumetric performance curves, $Q^{\prime \prime}$ ', depend almost exclusively on the surface area density $\alpha$. .

Figure 9 shows the following:

1. At a given $S_{t}$, a decrease in $S_{1}(J-B, E ; J-C, D)$ either decreases $Q{ }^{\prime} '$ at a given value of $\theta$ or increases $\theta$ for the same $Q^{\prime \prime}$ and $G_{\text {face }}$.

*Standard conditions used in this study were dry air at l atm and $500^{\circ} \mathrm{F}$. 


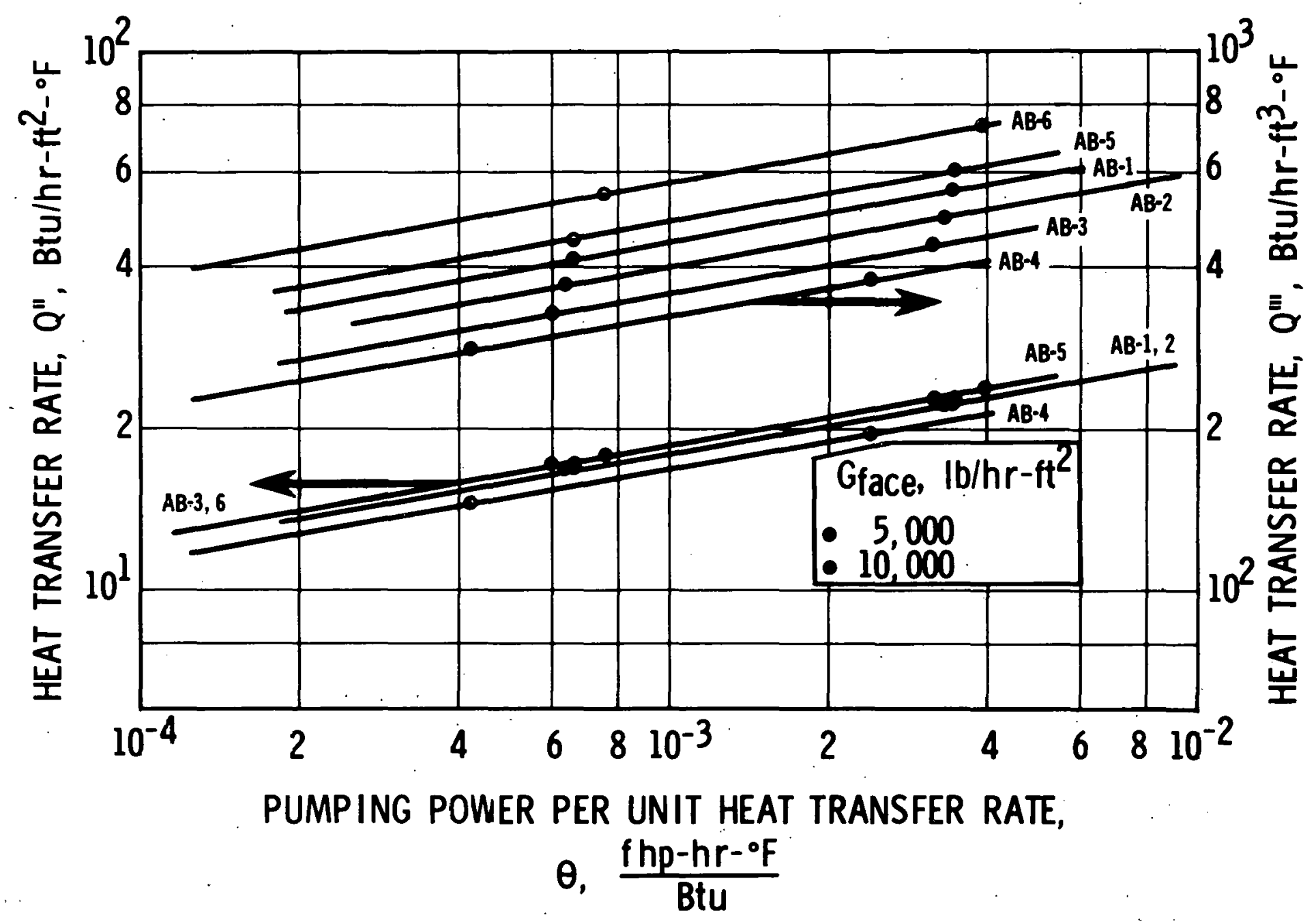

Figure 8

HEAT TRANSFER RATES FOR TUBE BANKS AB-1, 2, 3, 4, 5, 6

DESIGNATIONS FROM TABLE B-1 


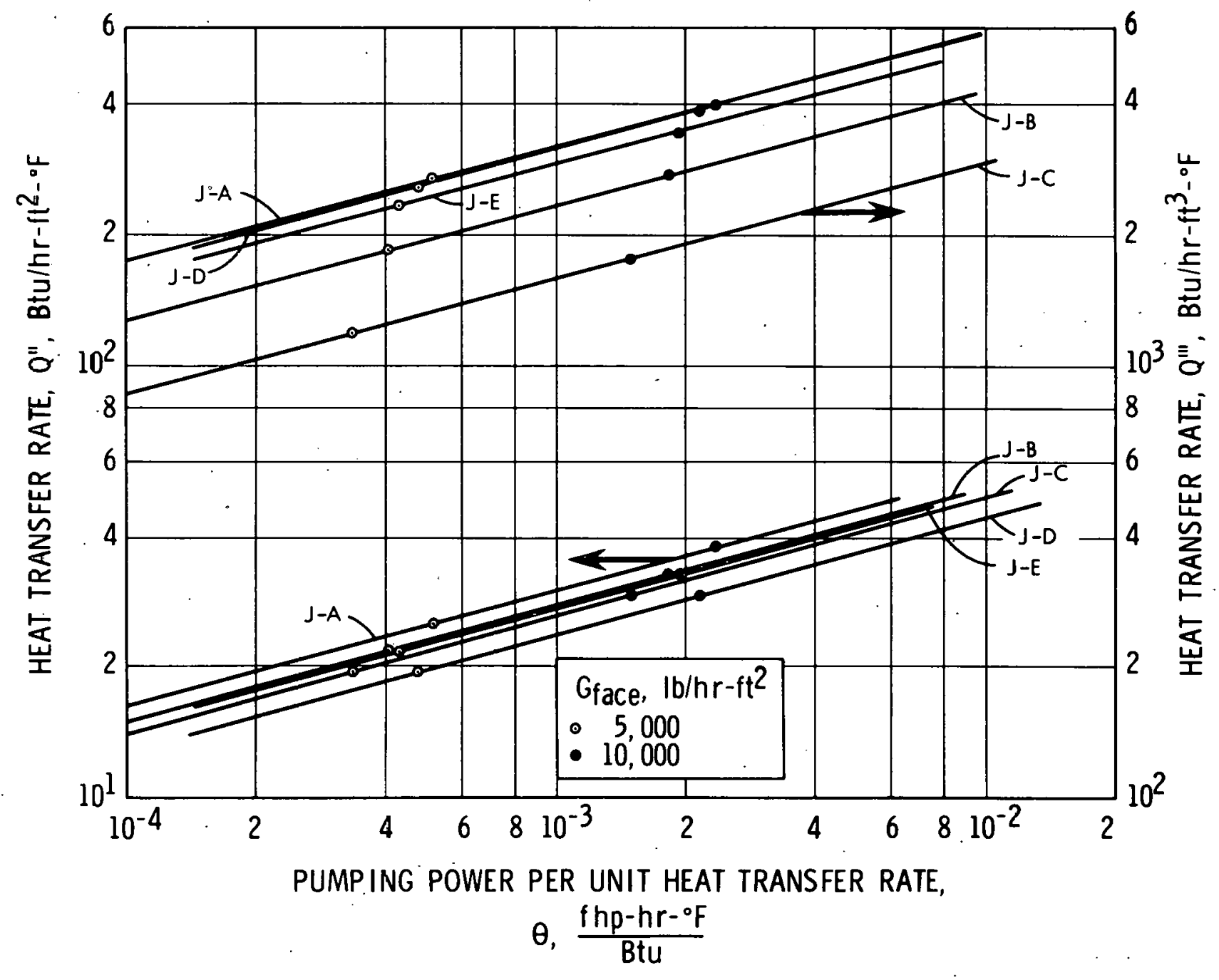

Figure 9

HEAT TRANSFER RATES FOR TUBE BANKS J-A, B, C, D, E DESIGNATIONS FROM.TABLE B-1 
2. When $S_{l}$ is held constant, decreasing $S_{t}(J-A, B, C)$ either increases $Q^{\prime \prime}$ and lowers $G_{\text {face }}$ at a given $\theta$, or increases both $Q^{\prime \prime}$ and $\theta$ at a given $G_{\text {face }}$.

3. The relative performance of the only tube tank with the minimum flow area in the diagonal spacings, $J-D$, was poor.

4. The volumetric performance curves, $Q$ ''', were not strictly dependent on $\alpha$. Arrangement $J-A$ transferred more heat than $J-D$, even though the latter had a' higher $\alpha$.

Figure 10 shows the two close packed staggered arrangements (COX $-2,3)$ to be higher performance than the widely spaced COX-1. The equilateral triangle arrangement, COX-2, appears to be slightly superior to the equivelocity COX-3. The in-line arrangement, COX-1, has lower levels of $Q^{\prime \prime}$ and $Q^{\prime \prime}$. At a given $G_{\text {face, }}$, however, $C O X-1$ has a much lower pumping power requirement.

The bare tube date in figure 11 shows that volumetric performance follows the same trend as the surface area density $\alpha$. On an area basis, relative performance often changes with increasing $\theta$. However, some trends are apparent:

1. If $S_{t}$ is held constant, a decrease in $S_{1}(K L-5,7,8)$ increases $Q^{\prime \prime}$ but not $\theta$ at a given $G_{\text {face }}$.

2. If $S_{\perp}$ is held constant, a decrease in $S_{t}(K L-5,6 ; K L-7,9)$ can increase $Q^{\prime \prime}$, but it also requires either a decrease in $\mathrm{G}_{\text {face }}$ or an increase in $\theta$.

3. In-line arrangements are low performance $(K L-13,14)$. Closer packed $K L-14$ requires a smaller $G_{\text {face }}$ for the same $Q^{\prime} '$.

4. There is no distinct effect from $A_{\min }$ being in the diagonal tube spacings $(K L-9,10)$. However, at the same $\alpha(K L-5,10)$ the tube bank with $A_{\min }$ in the diagonal spacings would require a smaller $G_{\text {face }}$ at equal values of $Q^{\prime \prime}$ and $\theta$.

The preceding comments indicate the tradeoffs required among heat transfer area, volume, pumping power, and frontal area in a final tube bank design. Close packed tube arrangements generally had higher curves for $Q^{\prime \prime}$ and $Q^{\prime \prime}$ '. This corresponds to lower heat transfer area and volume requirements. If there existed no restrictions on frontal areas, then those curves would probably indicate the desired tube arrangements. However, if frontal area were fixed, the selection of best pitch might become more difficult. An arrangement with a lower performance curve might then save enough in pumping power to offset its increased area or volume requirement.

In general, the statements made thus far should be applicable for turbulent flow through most tube banks. However, the effect that tube bank depth has on performance was neglected. Neal and Hitchcock ${ }^{8}$ found row-to-row heat transfer for finned tubes to vary considerably with the tube pitch. 


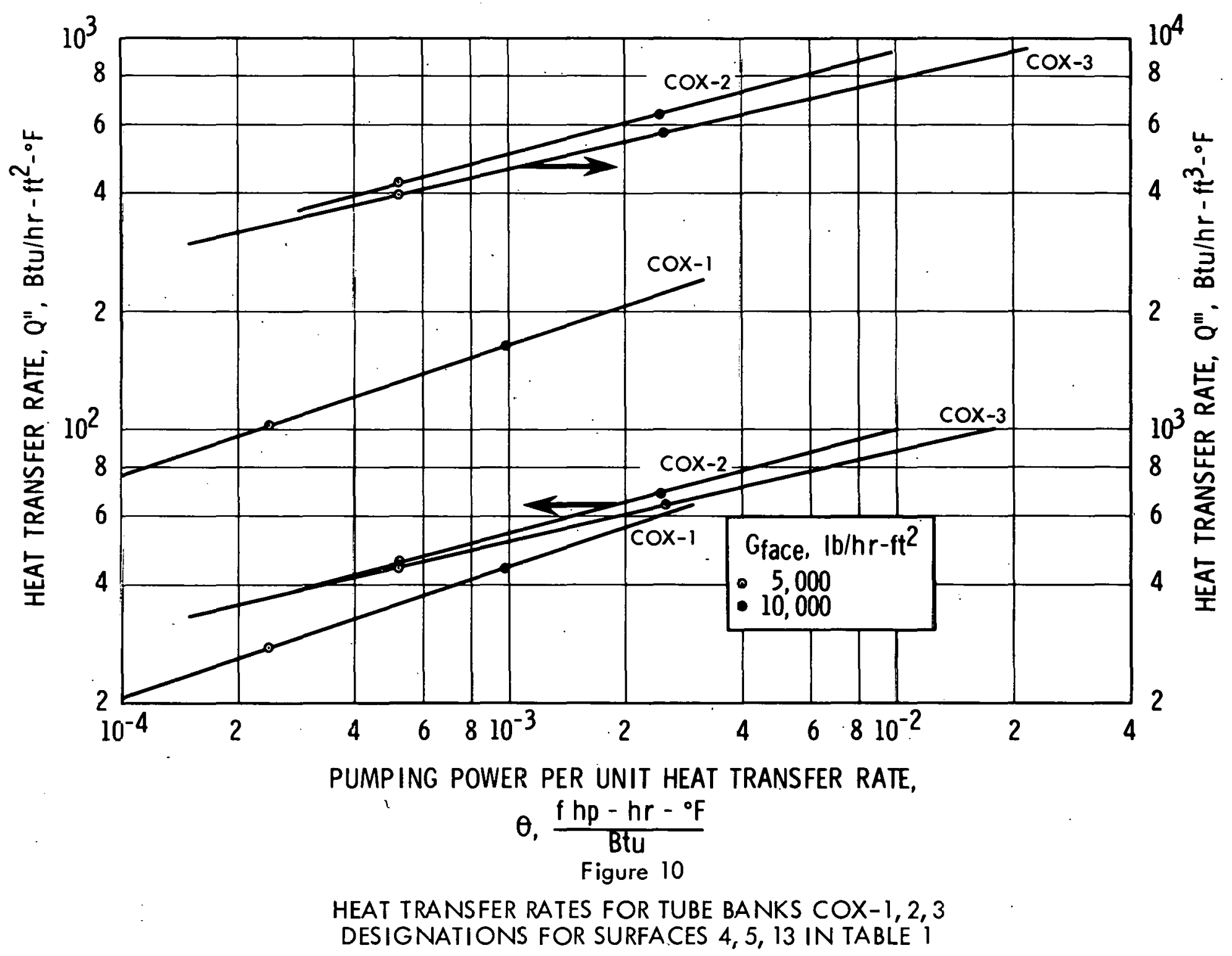




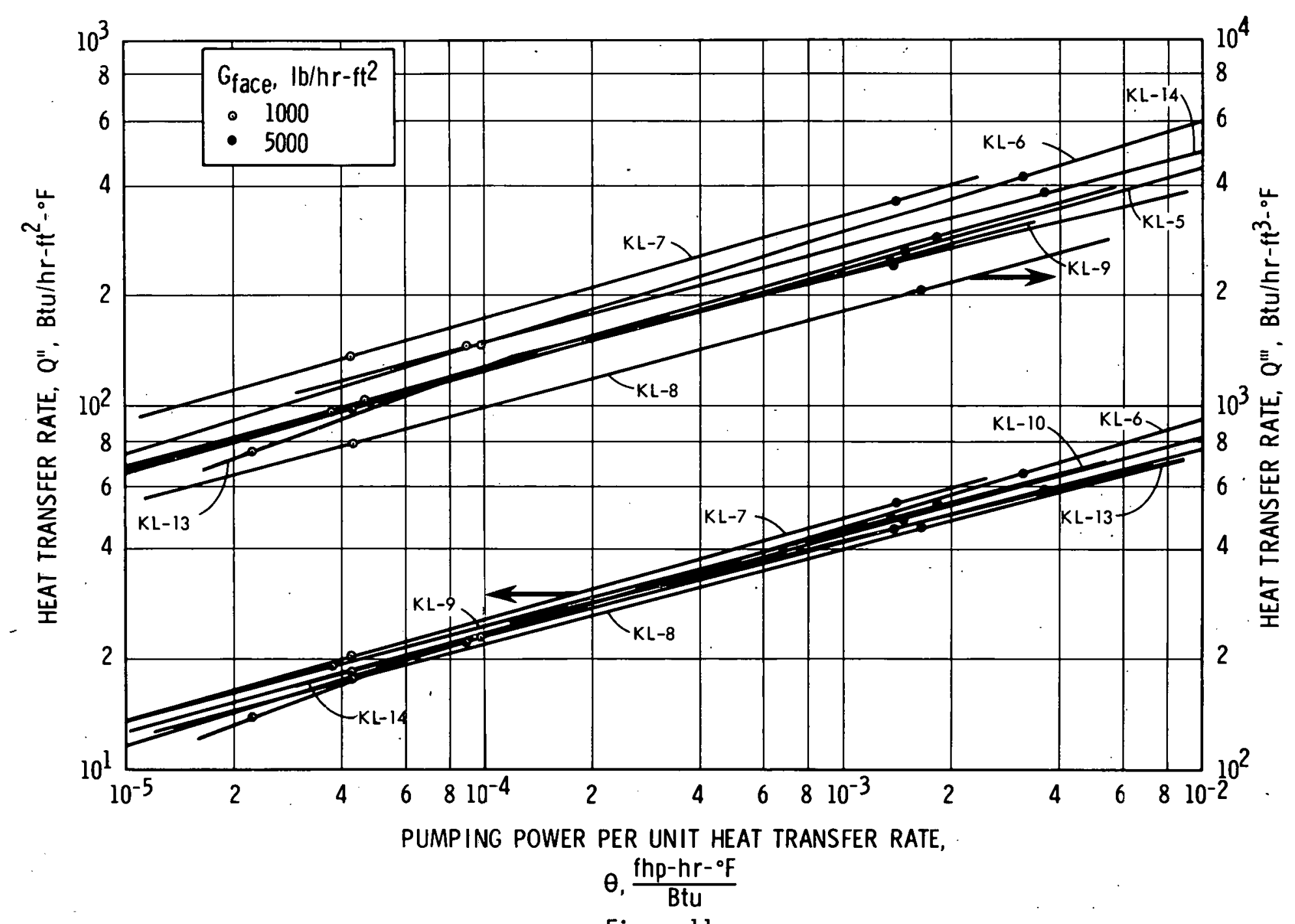

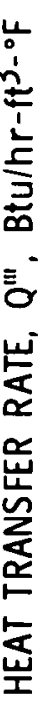

HEAT TRANSFER RATES FOR TUBE BANKS KL-5, 6, 7, 8, 9, 10, 13, 14 DESIGNATIONS FROM TABLE $B-2$ 
Kays, London, and $\mathrm{LO}^{9}$ found the row-to-row effects for bare tubes to vary only mildly with flow rate and pitch. Since pressure drop also exhibits row-to-row variations, 10 designers of shallow tube banks should exercise due caution.

\section{COMMENTS}

Several of the core samples that were tested had geometries or data results that are worth noting. One core sample emitted high-frequency noises.

The core sample designated surface 8 was retested several times in an attempt to resolve the anomalous heat transfer behavior discussed in reference 2 . It had been felt that tube side fouling resulted in the low heat transfer coefficients. However, numerous acid cleanings failed to improve the heat transfer rate. New headers to improve flow distribution also had no effect. Similarly, surface 9 appeared to exhibit poor heat transfer behavior. Several possible answers can be offered to at least partially explain this.

Both surface 8 and surface 9 were in-line tubes expanded onto plate fins. An obvious possibility is the existence of an appreciable gap or bond resistance between fin and tube. A less obvious explanation is the inherent error in the method of data reduction.

For purposes of data reduction, it was assumed ${ }^{2}$ that heat transfer coefficients were always uniform over the entire fin surface. Flow studies ${ }^{8}$ in tube banks similar to surface 6 have shown considerable variation in heat transfer coefficients and the existance of dead and reverse flow zones. With in-line tubes such as surfaces 8 and 9 these effects might be expected to become even more significant. The effects of heat transfer area on computed j-lines can be explained as follows.

Values for $h$ come from $\varepsilon h$ which depend upon $U$ which, in turn, varies inversely with heat transfer area. Plots of $h$ versus $\varepsilon h$ are not linear. In fact, the slope increases as $\varepsilon h$ increases. Thus, the larger the numerical value for $\varepsilon h$, the greater the effect of slight changes on calculated $h$ and $j$. This means that data reduced from small overall U's give apparent $h$ 's and j's higher than those from higher overall U's for the same amount of dead heat transfer area. Whether experimental U's were high or low depended upon whether the gas used in the experiments had a high or low thermal conductivity.

The two surfaces with spot welded, serrated helical fins had poor fin-totube bonding and poor uniformity of geometry. These surfaces, 11 and 12 , had their data reduced and plotted using average geometric values and assuming no fin bond resistance.

Over the higher range of experimental Reynolds numbers, surface 13 emitted a high-frequency whistling noise. This same phenomena was reported earlier for surface 5 . In neither case did any irregularities appear in the data. However, inspection of the petal fins for these surfaces showed that they had been flattened and in some cases broken. It is not known at what fluid velucity this damage occurred. There was no degradation in performance over the several weeks of testing. 
CONCLUSIONS

1. Basic heat transfer and flow friction design data are presented for seven high-finned tube banks. Fanning friction factors and Colburn $j$-factors are tabulated in Appendix $A$ and plotted versus Reynolds number in figures 1 through 7 .

2. Based on the finned tube bank results displayed in figures 8, 9, and 10:

a. An equilateral triangle tube pitch is attractive based on surface heat transfer rates, but it requires a large frontal area for a given pumping power expenditure. A close-packed equilateral triangle pitch is attractive based on volumetric heat transfer rates.

b. In-line arrangements display a lower surface heat transfer rate, but at a given pumping power they can operate with smaller frontal areas than the staggered banks.

c. Decreasing the transverse tube spacing instead of the longitudinal spacing is more effective in achieving a high-performance, higharea density tube bank.

3. Based on the bare tube bank results in figure 11:

a. In-line tubes are low performance arrangements.

b. Decreasing the longitudinal tube spacing instead of the transverse spacing is more effective in achieving a high-performance, higharea density tube bank.

4. Since entrance effects vary with both the type of tube and the tube arrangement, design of shallow tube banks may be difficult.

5. The use of a fin efficiency, especially for plate finned tubes, is of questionable value. It can lead to serious errors, especially when trying to apply data from one fluid to another. 


\section{APPENDIX A}

Geometric and Experimental Data 


\section{THIS PAGE}

WAS INTENTIONALLY

LEFT BLANK 
APPENDIX A

GEOMETRIC AND EXPERIMENTAL DATA

Table A-l

CORE SAMPLE GEOMETRIC DATA

\begin{tabular}{|c|c|c|c|c|c|}
\hline & \multicolumn{5}{|c|}{ Surface Number } \\
\hline & 9 & 10 & 11 & 12 & 13 \\
\hline$k_{f}, B t u / h r-f t-{ }^{\circ} F$ & 1.19 & 96 & 119 & 119 & 96 \\
\hline$k_{w}, B t u / h r-f t-{ }^{\circ} F$ & 119 & 96 & 119 & 119 & 96 \\
\hline$L_{h}, f t$ & 1.094 & $7 . .458$ & 1.445 & 1.083 & 0.607 \\
\hline$I_{c}, f t$ & 1.00 & 1.00 & 1.00 & 1.00 & 1.00 \\
\hline$A_{\min , h}$, sq $f t$ & 0.483 & 0.802 & 0.514 & 0.707 & 0.631 \\
\hline$A_{\min }, c, s q f t$ & 0.151 & 0.0892 & 0.149 & 0.0386 & 0.0760 \\
\hline$A_{h}, s q f t$ & 195 & 174 & 115 & 31.0 & 54.8 \\
\hline$A_{c}, s q f t$ & 8.16 & 28.0 & 9.49 & 4.88 & 9.01 \\
\hline$A_{w}, s q f t$ & $9.64=$ & 24.05 & 11.3 & 6.74 & 11.2 \\
\hline Afront $\mathrm{sq} \mathrm{ft}$ & 1.00 & 1.083 & 1.083 & 1.083 & 1.083 \\
\hline$t, f t$ & 0.00733 & 0.00292 & 0.00659 & 0.00707 & 0.00464 \\
\hline No. Rows Wide & 5 & 10 & 6 & 7 & 9 \\
\hline No Rows Deep & 7 & 7 & 8 & 7 & 10 \\
\hline
\end{tabular}


SURFACE NUMBER

1

\begin{tabular}{|c|c|c|c|c|c|c|c|c|c|c|c|c|c|}
\hline RUN & $\begin{array}{l}\text { AIR IN } \\
\text { OEG }\end{array}$ & $\begin{array}{l}\triangle I R \text { OUT } \\
\text { OEG R }\end{array}$ & $\begin{array}{l}\text { H2O IN } \\
\text { OEG: }\end{array}$ & $\begin{array}{c}\text { H2O DUT } \\
\text { OEG } R \\
.\end{array}$ & $\begin{array}{l}\text { AIR MEAN } \\
\text { PQESSURE } \\
\text { DSFA }\end{array}$ & $\begin{array}{l}\text { CORE DP } \\
\text { IN. H2O }\end{array}$ & $\begin{array}{l}\text { AIR } \\
\text { FLOW } \\
\text { SCFM }\end{array}$ & $\begin{array}{l}\text { WATER } \\
\text { FLOW } \\
\text { GPM }\end{array}$ & $\begin{array}{l}\text { HFAT } \\
\text { TRANSFER } \\
\text { BTU/MIN }\end{array}$ & $\begin{array}{l}\text { AIR HEAT } \\
\text { TRANSFER } \\
\text { COEFF. } \\
\text { BTU/HR FT F }\end{array}$ & $\begin{array}{l}\text { AIR } \\
\text { REYNOLDS } \\
\text { NUMBER }\end{array}$ & 5 & J \\
\hline 1. & 660.9 & 589.7 & 570.5 & 572.1 & 2101 & 2.360 & 1134 & 128.0 & 1499.4 & 23.88 & 3543 . & 0.02659 & 0.00796 \\
\hline 2. & 662.9 & 588.3 & 570.7 & $572 . \mathrm{C}$ & 2092 & 1.960 & 1056. & 128.0 & 1461.1 & 23.65 & 3304. & 0.02522 & 0.00847 \\
\hline 3 & 666.0 & $58,5.5$ & 571.1 & 572.2 & 2087. & 1.350 & 842. & .131 .0 & 1259.7 & 20.60 & 2646. & 0.02764 & 0.00925 \\
\hline 4. & 667.3 & 583.9 & 571.1 & 572.3 & 2119 & 1.000 & 736. & 130.0 & 1141.8 & 18.93 & 2319 & 0.02731 & 0.00967 \\
\hline 5. & $664 . t$ & 584.6 & 570.9 & 572.0 & 2146. & 1.130 & 800 . & 130.0 & 1198.6 & 19.68 & 2517 & 0.02646 & 0.00531 \\
\hline 6. & 645.3 & 603.1 & 569.4 & 572.1 & 2132. & 14.360 & 3368 & 126.0 & 2638.0 & 51.74 & 10421 & 0.01817 & 0.00580 \\
\hline 7. & 647.5 & 602.1 & $5 \in 9.6$ & 572.0 & 2128 & 11.900 & 3045 . & 126.0 & 2566.9 & 49.52 & 9423. & 0.01844 & 0.90602 \\
\hline ค. & 649.0 & 599.7 & 569.8 & 572.0 & 2122 & 9.040 & 2583. & 126.0 & 2366.5 & 43.75 & 8008 & 0.01951 & 0.00640 \\
\hline 9. & 650.8 & 597.8 & 569.7 & 571.8 & 2116. & 7.120 & 2238 & 126.0 & 2203.8 & 39.28 & 6949 . & 0.02047 & 0.00663 \\
\hline 10. & 652.7 & 595.2 & 570.3 & 572.2 & 2103 & 4.980 & 1814. & 126.0 & 1937.5 & 33.69 & 5643 . & 0.02178 & 0.00702 \\
\hline 11. & $65^{2} \cdot t$ & 593.6 & 570.1 & 572.0 & $209 \theta$ & 4.120 & $162 ?$. & 126.0 & 1820.3 & 31.30 & 5086 & 0.02227 & 0.00724 \\
\hline 12. & 663.6 & 588.9 & 570.5 & 571.8 & 2144. & 1.520 & 953. & 126.0 & 1356.9 & 21.97 & 2987 . & 0.02495 & 0.00872 \\
\hline 13. & $65 \mathrm{i} .0$ & 597.2 & 569.2 & 571.8 & 2110 & 4.960 & 1816. & 82.0 & 1815.6 & 33.54 & 5639. & 0.02164 & 0.00698 \\
\hline 14. & 691.1 & 596.7 & $569: 6$ & 572.1 & 2110 & 4.960 & 1824. & 96.0 & 1943.5 & 33.02 & 5637. & 0.02148 & 0.00684 \\
\hline 15. & 653.3 & 595.7 & 570.2 & 572.2 & 2110 & 4.930 & $180^{\circ}$ & 118.5 & 1938.8 & 33.71 & 5624. & 0.02173 & 0.00704 \\
\hline 14. & 6.53 .9 & 594.5 & 570.4 & 572.1 & 2110 & 4.970 & 1827 & 160.0 & 2015.4 & 33.58 & 5688 . & 0.02152 & 0.00694 \\
\hline 17. & 654.5 & 593.5 & 570.8 & 572.1 & 2110 & 4.050 & 1829 & 212.0 & 2072.6 & 33.55 & 5699. & 0.02144 & 0.00693 \\
\hline 18. & 6.56 .2 & 592.6 & 570.8 & 571.9 & 2110 & .4 .970 & 1821. & 296.0 & 2151.5 & 37.45 & 5679. & 0.02174 & 0.00694 \\
\hline 19. & 657.6 & 591.8 & 570.6 & 572.1 & 2098. & 3.400 & 1492. & 158.0 & 1824.2 & 30.03 & 4653. & 0.02267 & 0.00761 \\
\hline 20. & 659.6 & 590.6 & 570.5 & 572.1 & 2094 & 3.010 & 1373. & 158.0 & 1760.3 & 28.77 & $428 x$. & 0.02309 & 0.00792 \\
\hline 21. & 666.1 . & 585.9 & 570.9 & & 2067. & & 858 & 158.0 & 1280.1 & 19.88 & 2694. & 0.02731 & \\
\hline $220^{\circ}$ & 669.9 & 523.8 & 571.1 & 572.2 & 2083. & 0.995 & 730 & 156.0 & 1169.3 & 18.46 & 2299. & 0.02718 & 0.00956 \\
\hline 23. & 670.6 & 593.2 & 571.0 & 572.1 & $208 E$. & c. 900 & 700. & 156.0 & 1137.4 & 18.02 & 2205. & 0.02684 & 0.00574 \\
\hline 24. & 650.0 & 648.9 & & $* * * *$ & 2175 & 10.970 & 2808 & \multicolumn{3}{|c|}{$0.0 * * * * * * * * * * * * 0 * * * * * *$} & 8393. & \multicolumn{2}{|c|}{$0.01916 * * 0 * * * * *$} \\
\hline 25. & $\$ 50.0$ & 649.7 & & & $213 \geq$. & 7.120 & $215 \theta$. & \multicolumn{3}{|c|}{$0.0 * * * * * * * * * * * * * * * * * *$} & 6449. & \multicolumn{2}{|c|}{$0.02072 * * * * * * * * 4$} \\
\hline 26. & 650.0 & & & & 2107. & & 1588. & \multicolumn{3}{|c|}{$0.0 * * * * * * * * * * * * * * * * * *$} & 4748. & \multicolumn{2}{|c|}{$0.02258 * * * * * * * * *$} \\
\hline 27. & 650.0 & & & & & & 1041. & \multicolumn{3}{|c|}{$0.0 * * * * 0 * * * * \dot{*} * 0 * * * * * * * *$} & 3113 & $0.02531 \%$ & $* * * * * * * *$ \\
\hline 8. & 649.8 & 667.7 & & 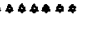 & 2085. & 1.520 & 883. & $0.0 *$ & 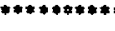 & $* * * * * *$ & 2640. & \multicolumn{2}{|c|}{$0.02595 * * * * * * 4 * *$} \\
\hline
\end{tabular}


SURFACE NUMBER 8

\begin{tabular}{|c|c|c|c|c|c|c|c|c|c|c|c|c|c|}
\hline RUN & $\begin{array}{l}\text { AIR IN } \\
\text { DEG R }\end{array}$ & $\begin{array}{l}\text { BIR OUT } \\
\text { DEG Q }\end{array}$ & $\begin{array}{l}\text { H20 IN } \\
\text { DEG }\end{array}$ & $\begin{array}{c}\text { H2O OUT } \\
\text { DEG } R\end{array}$ & $\begin{array}{l}\text { AIR MEAN } \\
\text { PRES SURE } \\
\text { PSFA }\end{array}$ & $\begin{array}{l}\text { CORE DP } \\
\text { IN. H2O }\end{array}$ & $\begin{array}{l}\text { AIR } \\
\text { FLOW } \\
\text { SCFM }\end{array}$ & $\begin{array}{l}\text { MATER } \\
\text { FLDW } \\
\text { GPF }\end{array}$ & $\begin{array}{c}\text { HEAT } \\
\text { TRANSFER } \\
\text { RTUIMIN }\end{array}$ & $\begin{array}{l}\text { AIR HEAT } \\
\text { TRANSFER } \\
\text { COEFF. } \\
\text { BTU/HA FT F }\end{array}$ & $\begin{array}{c}\text { AIR } \\
\text { REYNDLDS } \\
\text { NUMBER }\end{array}$ & $s$ & $J$ \\
\hline 1. & 652.7 & $651.6 * *$ & $* * * * * * * * *$ & $* * * * * * 0$ & 2185. & 12.100 & 2758. & 0.0 & $0.0 * *$ & $* * * * * * * * *$ & 4033. & $0.00676 * 8$ & $* * * * * * * * *$ \\
\hline 2. & 652.7 & $651.4 *$ & 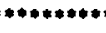 & $* * * * * * *$ & 2148. & 7.120 & 2009. & 0.0 & $0.0 * *$ & $* * * * * * * * * *$ & 2938 . & $0.00740 *$ & $* * * * * * * * *$ \\
\hline 3. & 653.2 & $651.5 * 1$ & $* * * * * * * * *$ & $* * * * * * \bullet$ & 2131. & 4.740 & 1549. & 0.0 & $0.0 * *$ & $* * * * \forall * * * *$ & 2265. & $0.00822 *$ & $* * * *$ \\
\hline 4. & 653.8 & $652.9 * 4$ & 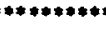 & 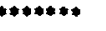 & 2118. & 3.130 & 1103. & 0.0 & $0.0 * *$ & $* * * * * * * * *$ & 1743. & $0.00909 *$ & $* * * * * * * * *$ \\
\hline 5. & 654.4 & $652.1 * 4$ & $* * * * * * * *$ & $* * * * * * *$ & 2111 & 1.980 & 863. & 0.0 & $0.0 * *$ & $* * * * * * * * *$ & 1260. & $0.01097 *$ & $* * * * * * * * *$ \\
\hline 6. & 628.0 & 607.7 & 569.7 & 571.7 & 2218 & 16.620 & 3438. & 121.0 & 1936.9 & 20.79 & 5216. & 0.00644 & 0.00203 \\
\hline 7. & 638.8 & 605.1 & 570.1 & 571.9 & 2168 & 12.270 & 2831 & 120.5 & 1775.0 & 18.12 & 4305 & 0.00689 & 0.00215 \\
\hline 8. & $B 40.3$ & 601.4 & 570.4 & 572.0 & 2126. & 7.930 & 2155. & 120.5 & 1557.1 & 14.84 & 3286. & 0.00759 & 0.00231 \\
\hline 9. & 644.3 & 597.0 & 570.7 & 572.0 & 2154 & 4.150 & 1505. & $120.5^{\circ}$ & 1323.1 & 11.45 & 2301 . & 0.00832 & 0.00256 \\
\hline 10. & 647.2 & 503.2 & 570.4 & 571.7 & 2151. & 2.750 & .1172 & 120.5 & 1176.9 & 9.84 & 1707. & 0.00914 & 0.00283 \\
\hline 11. & 651.6 & 587.5 & 570.7 & 571.7 & 2104 & 1.610 & .843. & 120.5 & 1004.9 & 8.70 & 1301. & 0.01020 & 0.00348 \\
\hline 12. & 638.1 & 607.1 & 589.7 & 571.7 & 2225. & 10.150 & 3388 & 119.5 & 1252.8 & 22.07 & 5145. & 0.00647 & 0.00219 \\
\hline 13. & 639.0 & 605.2 & $570 . \mathrm{C}$ & 571.9 & 2211. & 12.400 & 2891. & 120.0 & 1819.1 & 19.17 & 4395. & 0.00881 & 0.00223 \\
\hline 14. & 639.4 & 603.1 & 569.8 & 571.7 & 2178. & 9.880 & 2540. & 120.0 & 1714.4 & 17.49 & 3869. & 0.00696 & 0.00232 \\
\hline 15. & 641.3 & 601.6 & 570.3 & 572.0 & 2170. & 7.570 & 2160 & 120.0 & 1591.1 & 15.22 & 3201 . & 0.00737 & 0.00237 \\
\hline 16. & 644.5 & 598.6 & 570.2 & 571.7 & 2159. & 5.040 & 1683. & 120.0 & 1434.2 & 12.60 & 2569. & 0.00807 & 0.00252 \\
\hline 17. & 638.0 & 007.8 & 369.4 & 571.7 & 2226. & 15.850 & 3356 & 114.0 & 1885.8 & 20.15 & 5094 . & 0.00647 & 0.00202 \\
\hline 18. & $B 38.1$ & 200.4 & 569.4 & 571.6 & 2205. & 13.980 & 3090 . & 113.5 & 1916.8 & 19.16 & 4696 . & 0.00668 & 0.00208 \\
\hline 19. & 638.2 & 604.4 & 570.5 & 572.3 & 2180 & 10.960 & 2674. & 115.0 & 1679.2 & 17.40 & 4071 . & 0.00695 & 0.00219 \\
\hline 20. & 641.2 & 603.8 & 570.1 & 571.9 & 2184 & 9.050 & 2386. & 115.0 & 1657.5 & 15.91 & 3028 . & 0.00723 & 0.00224 \\
\hline 21. & $t 41.1$ & 602.7 & 570.4 & 572.1 & $21 \leqslant 0$ & 8.050 & 2221. & 115.0 & 1585.1 & 15.09 & 3382 . & 0.00736 & 0.00228 \\
\hline 22 . & 643.0 & 509.6 & 570.2 & 571.8 & 2131. & 6.040 & 1871. & 126.0 & 1509.4 & 13.58 & 2855 . & 0.00772 & 0.00244 \\
\hline 23. & 644.0 & 595.9 & 570.2 & 571.5 & 2116. & 4.010 & 1464 & 126.0 & 1332.6 & 11.36 & 2240. & 0.00836 & 0.00261 \\
\hline 24. & 654.4 & 595.8 & 570.0 & 571.3 & 2136. & 3.070 & 1262. & $126.0^{\circ}$ & 1375.9 & 10.53 & $19 ? 5$. & 0.00868 & 0.00281 \\
\hline 25. & 657.1 & 591.4 & 570.8 & 571.9 & 2112. & 2.100 & 972. & 126.0 & 1187.0 & 9.27 & 1490 . & 0.00997 & 0.00321 \\
\hline PA. & 640.2 & 605.0 & 569.8 & 571.3 & 2153. & 15.950 & 3316. & 174.0 & 2169.9 & 21.59 & 5040. & 0.00647 & 0.00219 \\
\hline 27. & 841.0 & 602.6 & 570.6 & 572.0 & 2146. & 11.090 & 2661. & 174.0 & 1929.2 & 17.96 & 4051 . & u.uoruu & 0.00227 \\
\hline 28. & 643.5 & 599.2 & 70.8 & 72 & 2128. & 7.120 & 2069. & 174.0 & 1702.4 & 14.98 & 3158. & 0.00743 & 0.00244 \\
\hline 29. & 645.3 & 594.7 & 571.3 & 572.2 & $216 \mathrm{c}$ & 4.050 & $1491^{\circ}$ & 174.0 & 1400.6 & 11.70 & 2284. & 0.00834 & 0.00204 \\
\hline 30. & 639.8 & 600.6 & 590.1 & 572.0 & 2158. & 5.918 & 1078 & 89.0 & 1367.1 & 14.08 & 2867. & 0.00768 & 0.00252 \\
\hline 31. & 640.2 & 599.8 & 570.3 & 572.0 & 2158. & 5.995 & 1885 & 105.0 & 1413.6 & 13.90 & 2879 . & 0.00764 & 0.00246 \\
\hline 32. & 641.5 & 599.6 & 570.4 & 571.9 & 2162. & .997 & 1888. & 118.0 & 1470.9 & 13.80 & 28 ค2. & 0.00764 & 0.00246 \\
\hline 33. & $04<.5$ & $3 \div 0: 7$ & 570.5 & 571.0 & lnas. & 5.997 & 1859. & 139.0 & 1510.1 & 13.45 & 2838 . & 0.00790 & 0.00244 \\
\hline 34. & 0.42 .1 & 597.6 & 570.9 & 572.0 & 2162. & 6.003 & 1890 & 169.0 & 1598.2 & 14.18 & 28 ค०, & 0.00766 & 0.00252 \\
\hline 35. & 643.9 & 500.7 & 971.2 & 572.1 & 2162. & B. ก09 & 1891. & 222.0 & 1658.0 & 13.94 & 2893. & 0.00766 & $0 . J 0248$ \\
\hline 36. & 644.8 & 596.8 & 570.5 & 571.7 & $210 t$. & 4.990 & 1660. & 150.0 & 1474.85 & 12.71 & $253 n$. & 0.00804 & $0.0 n 258$ \\
\hline 37. & 096.6 & 592.4 & 570.5 & 571.5 & 2009. & 2.520 & 1085 & 150.0 & 1293.9 & 9.65 & 1661. & 0.00948 & 0.00299 \\
\hline 38. & 659.8 & 589.0 & 570.5 & 571.5 & 2115. & 1.860 & 896. & 150.0 & 1178.9 & 8.92 & 1377 & 0.01044 & 0.00335 \\
\hline 39. & 643.1 & 596.2 & 570.7 & & 2132. & & 1460. & 124.0 & 1273.1 & 10.94 & 2236 & 0.00836 & 0.00252 \\
\hline 40. & 644.1 & 593.7 & 570.6 & 571.7 & 2127. & 3.090 & 1261. & 124.0 & 1182.1 & 10.11 & 1935. & 0.00877 & 0.00270 \\
\hline 41. & 689.7 & 629.4 & 573.2 & 576.0 & 2138. & 11.070 & 2576. & 124.0 & 2891.4 & 16.87 & 3758. & 0.00708 & 0.00219 \\
\hline 42. & 602. & h?h.B & 572.9 & & & & & & 2756.3 & & 3335. & 0.00725 & 0.00231 \\
\hline 43. & 895.3 & 621.6 & 573.2 & 575.8 & 2120. & & 1814 & 124.0 & 2488.4 & & 2659. & 0.00807 & 0.00245 \\
\hline 44. & 699.8 & 616.2 & 573.4 & 575.7 & 2109 & 4.310 & 1439 & 124.0 & 2230.4 & 11.38 & 2116 & 0.00869 & \\
\hline 45. & 706.7 & 606.7 & 574.7 & 576.2 & 2100 & & 1001 & 124.0 & 1863.0 & & 1485 & 0.00995 & 0.00320 \\
\hline 46. & 707.4 & & 574.5 & 575.7 & 2164. & & 830. & 124.0 & 1653.7 & 8.90 & 1241 . & 121 & 360 \\
\hline 47. & 650.2 & 589.7 & 571.0 & $572 . \mathrm{C}$ & & 2.000 & 951. & 124.0 & 1069.0 & 9.06 & 1463. & 0.01012 & 0.00321 \\
\hline 48. & & & 570.1 & 571.4 & 21 & 2.510 & 1108 & 124.0 & 1160.5 & 9.35 & 1699. & 0.00931 & 0.00284 \\
\hline & & & & & & & & & 1 & & 364. & 651 & 00213 \\
\hline
\end{tabular}


SUP.FACE NUMBEP,

\begin{tabular}{|c|c|c|c|c|c|c|c|c|c|c|c|c|c|}
\hline RUN & $\begin{array}{l}\text { AIP IN } \\
\text { DEG R }\end{array}$ & $\begin{array}{l}\triangle \text { IR OUT } \\
\text { OEG R }\end{array}$ & $\begin{array}{l}\text { H2O IN } \\
\text { DEG R }\end{array}$ & $\begin{array}{l}\text { HOR DUT } \\
\text { DEG R }\end{array}$ & $\begin{array}{l}\text { A IR NEAN } \\
\text { PRES SURE } \\
\text { PSFA }\end{array}$ & $\begin{array}{l}\text { CORE DP } \\
\text { IN. H2O }\end{array}$ & $\begin{array}{l}\text { AIR } \\
\text { FLOW } \\
\text { SCFM }\end{array}$ & $\begin{array}{l}\text { WATER } \\
\text { FLOW } \\
\text { GDM }\end{array}$ & $\begin{array}{c}\text { HFAT } \\
\text { TRANSFER } \\
\text { BTU/MIN }\end{array}$ & $\begin{array}{l}\text { AIR HEAT } \\
\text { TRANSFER } \\
\text { COEFF. } \\
\text { OTU/HR FT F }\end{array}$ & $\begin{array}{l}\text { AIR } \\
\text { REYNDLOS } \\
\text { NUHBER }\end{array}$ & 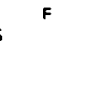 & $J$ \\
\hline 1. & 635.8 & 608.2 & 570.3 & 572.1 & 2211. & 18.980 & 3013. & 119.5 & 1545.1 & 18.74 & 6215. & 0.01653 & 0.00214 \\
\hline 2. & 635.4 & 605.0 & 570.5 & 572.1 & 2188. & 14.160 & 2543. & 119.5 & 1438.7 & 17.95 & 5261 . & 0.01724 & 0.00243 \\
\hline 3. & 636.0 & 601.2 & 570.7 & 572.3 & $217 c$ & 9.100 & 1984. & 119.5 & 1314.9 & 16.64 & 4116. & $0.0 ! 818$ & 0.00288 \\
\hline 4. & 638.5 & 595.7 & 570.5 & 571,8 & 2157. & 5.100 & 1437. & 119.5 & 1142.3 & 14.70 & 2995. & 0.01947 & 0.00352 \\
\hline 5. & 640.3 & 591.8 & 570.8 & 572.1 & 2150 & 3.300 & 1094. & 119.5 & 985.5 & 12.85 & 2288. & 0.02181 & 0.00404 \\
\hline B. & 662.7 & 588.5 & $=70.0$ & 572.2 & 2124. & 1.660 & 755 & 119.8 & 1041.0 & 11.90 & 1575 . & 0.02279 & 0.00542 \\
\hline 7 . & 639.9 & 599.2 & 571.2 & 572.1 & 2170 & 8.740 & 1931. & 307.0 & 1461.3 & 15.68 & 4009 & 0.01847 & 0.00279 \\
\hline e. & 639.8 & 599.8 & 571.2 & 572.2 & 2169 & 8. 730 & $193 \%$ & 251.0 & 1439.6 & 15.72 & 4020 & 0.01830 & 0.00279 \\
\hline 9. & 640.1 & 601.0 & 570.8 & 571.9 & 2169. & 8.730 & 1937. & 202.0 & 1406.9 & 15.22 & 4013. & $0.019 ? 9$ & 0.00270 \\
\hline o. & 639.6 & 601.2 & 570.6 & 571.9 & 2168. & e. 720 & 1936. & 162.0 & 1382.9 & 15.63 & 4011 & 0.01828 & 0.00278 \\
\hline 11. & 639.3 & 601.8 & 570.5 & 571.0 & 2168. & 8.730 & 1935. & 133.0 & 1349.8 & 15.74 & 4007 & 0.01830 & 0.00280 \\
\hline 12. & 638.9 & 602.8 & 570.4 & 572.1 & 2168. & 8.750 & 1933. & $11 ? .0$ & 1294.4 & 15.31 & 4001 . & 0.01834 & 0.00272 \\
\hline 13. & 638.8 & 603.6 & 570.2 & 572.0 & 2147. & 8.740 & 1932. & 90.0 & 1263.3 & 15.67 & 3996. & 0.01814 & 0.00279 \\
\hline 14. & 645.3 & 593.1 & 570.5 & 571.5 & 2105. & 3.250 & 1097 & 120.0 & $1062 \cdot 8$ & 12.77 & 2286 & 0.02090 & 0.00401 \\
\hline 15. & 635.5 & 608.2 & 570.2 & 572.0 & 2219. & 18.900 & 2994. & 120.0 & 1516.7 & 18.07 & 6178. & 0.01673 & 0.00207 \\
\hline 16. & 634.0 & $6 C 5.2$ & 570.1 & 572.2 & 2163. & 11.620 & 2260 & 70.0 & 1245.7 & 17.81 & 4676. & 0.01772 & 0.00271 \\
\hline 17. & 636.2 & 600.8 & 570.2 & 577.1 & 2136 & 7.170 & 1726. & 70.0 & 1135.4 & 16.39 & 3582 . & 0.01866 & 0.00327 \\
\hline 1A, & $6<3.1$ & 597.8 & 570.3 & 572.1 & $211 \mathrm{e}$ & 4.130 & 1264. & 70.0 & $10 \notin 4.1$ & 14.23 & 2625 . & 0.01992 & 0.00387 \\
\hline 9. & 645.3 & 503.0 & 570.5 & 572.2 & 2125. & 2.540 & 950. & 70.0 & 933.0 & 12.69 & 2001 . & 0.02153 & 0.00455 \\
\hline 20. & 648.2 & 570.1 & 571.0 & 572.0 & 2163. & 0.760 & 468. & 100.0 & 601.0 & 9.68 & 996. & 0.02823 & 0.00712 \\
\hline 21. & 643.9 & 582.8 & $570 . P$ & 571.9 & 2164. & 1.220 & 635. & 100.0 & 721.1 & 11.06 & 1344. & 0.02445 & 0.00600 \\
\hline 22. & 630.8 & $5 A 6.6$ & 570.6 & 571.8 & 2169. & 1.870 & 825. & 100.0 & 814.4 & 11.95 & 1737 & 0.02214 & 0.00499 \\
\hline 23. & 623.4 & 602.9 & 570.5 & $572 . ?$ & $210 \%$ & 20.700 & 3185. & 100.0 & 1212.9 & 18.37 & 6638. & 0.01553 & 0.00198 \\
\hline 24. & 675.7 & $601 . ?$ & 570.2 & 571.8 & 2182. & 14.760 & 2616. & 100.0 & 1194.7 & 17.43 & 5454. & 0.01707 & 0.00229 \\
\hline 25. & 627.4 & 509.9 & 570.5 & 572.1 & 2159. & 11.040 & 2200 & 100.0 & 1126.5 & 16.24 & 4587. & 0.01792 & 0.00254 \\
\hline 26. & 636.9 & & & netests & & & & & \multicolumn{2}{|c|}{$0.0 * * * * * * * * * *$} & & & \\
\hline 27. & 637. & & & * & & & & 0.0 & \multicolumn{2}{|c|}{$0.0 * * * * * * * * * * *$} & & & \\
\hline 28. & 637.2 & $636.1 * 0$ & & 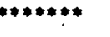 & 2125 & 7.470 & 1713. & $n . n$ & \multicolumn{2}{|c|}{ 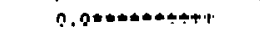 } & 9405: & 0.0181 & \\
\hline 29. & 637.4 & & & & & 5.100 & 1370. & 0.0 & \multicolumn{2}{|c|}{$0.0 * * * * * * * * * *$} & 2797. & $0.01971 *$ & \\
\hline & 639.7 & & & & & 2.800 & 991. & 0.0 & \multicolumn{2}{|c|}{$0.0 * * * * * * * * * * *$} & 1939. & $0: 02$ & \\
\hline 1. & 641.8 & $639.5 * 4$ & $* * * * * * * *$ & $* * * * * * *$ & 2097 . & 2.660 & 949. & 0.0 & \multicolumn{2}{|c|}{$0.0 * * * * * * * * * * *$} & 1909. & 0.02 .1474 & $* 4 * * * 4 * *$ \\
\hline
\end{tabular}


SURFACE NUMBER 10

\begin{tabular}{|c|c|c|c|c|c|c|c|c|c|c|c|c|c|}
\hline RUN & $\begin{array}{l}\text { AIR IN } \\
\text { OEG }\end{array}$ & $\begin{array}{l}\text { AIR OUT } \\
\text { DEG R }\end{array}$ & $\begin{array}{l}\text { H2O IN } \\
\text { DEG } R\end{array}$ & $\underset{\text { DEG } R}{\text { H2O OUT }}$ & $\begin{array}{l}\text { AIR MFAN } \\
\text { PRES SURE } \\
\text { PSFA }\end{array}$ & $\begin{array}{l}\text { CORE OP } \\
\text { IN. H2O }\end{array}$ & $\begin{array}{l}\text { AIR } \\
\text { FLDW } \\
\text { SCFM }\end{array}$ & $\begin{array}{l}\text { WATER } \\
\text { FLOW } \\
\text { GPY }\end{array}$ & $\begin{array}{l}\text { MEAT } \\
\text { TRANSFER } \\
\text { BTUSMIN }\end{array}$ & $\begin{array}{l}\text { AIR HEAT } \\
\text { IRANSFER } \\
\text { COEFF } \\
\text { BTUIHR FT F }\end{array}$ & $\begin{array}{l}\text { AIR } \\
\text { REYNOLDS } \\
\text { NUMBER }\end{array}$ & s & $J$ \\
\hline 1. & 641.9 & 594.5 & 570.9 & 572.6 & 2079. & 6.269 & 3705 . & 250.0 & 3264.5 & 40.53 & 11571. & 0.01812 & 0.00624 \\
\hline 2. & 643.0 & 594.3 & 570.4 & 572.2 & 2080 & 6.001 & 3639. & 250.0 & 3289.5 & 40.02 & 11361 . & 0.01801 & 0.00628 \\
\hline 3. & 643.2 & 593.5 & 570.3 & 571.9 & 2083. & 5.489 & 3455 . & 250.0 & 3187.6 & 38.73 & 10796 & 0.01833 & 0.00640 \\
\hline 4. & 643.0 & 593.9 & 571.4 & $573 . \mathrm{C}$ & 2086 & 5.052 & 3291 . & 250.0 & 3004.8 & 36.91 & 10282 & 0.01962 & 0.00640 \\
\hline 5. & 643.8 & 592.7 & 570.8 & $572 \cdot 3$ & 2090 & 4.503 & 3116. & 250.0 & 2958.1 & 36.02 & 9743. & 0.01860 & 0.00660 \\
\hline 6. & 644.6 & $592 . ?$ & 570.4 & 572.0 & 2091 . & 4.202 & 2999. & 250.0 & 2915.3 & 35.08 & 9351. & 0.01889 & 0.00670 \\
\hline 7. & 645.4 & 591.9 & 570.3 & 571.8 & 2092 & 3.924 & 2068. & 250.0 & 2850.7 & 33.85 & 8971 . & 0.01919 & 0.00674 \\
\hline 8. & 644.4 & 590.9 & 570.6 & 572.0 & 2107 & 3.442 & 2658. & 249.0 & 2642.3 & 31.98 & 8325. & 0.01978 & 0.00687 \\
\hline$\circ$ & 645.5 & 590.0 & 570.9 & 572.3 & 2108 & 3.212 & 2555 . & 249.0 & 2592.7 & 31.18 & 8002. & 0.01998 & 0.00697 \\
\hline 10. & 645.2 & 590.3 & 570.7 & 572.0 & 2109 & 2.993 & 2475 & 249.0 & 2526.3 & 30.44 & 7757. & 0.01988 & 0.00703 \\
\hline 11. & 645.5 & 590.5 & 571.1 & 572.4 & 2109. & 2.766 & 2391 & 249.0 & 2440.6 & 29.26 & 7493 & 0.01969 & 0.00699 \\
\hline 12. & 645.6 & 540.4 & 570.7 & 572.0 & 2110 & 2.623 & 2358. & 240.0 & 2419.3 & 2.1 .62 & 7390. & 0.01922 & 0.00693 \\
\hline 13. & 645.9 & 590.3 & 570.7 & $57 ? .0$ & 2111. & 2.402 & 2255 & 249.0 & 2328.6 & 27.13 & 7065. & 0.01927 & 0.00687 \\
\hline 14. & 646.0 & 590.2 & 571.2 & 572.5 & 2112 & 2.226 & 2165. & 249.0 & 2241.6 & 26.33 & 6786. & 0.01938 & 0.00695 \\
\hline 15. & 645.7 & 589.9 & 571.2 & 572.4 & 2085 & 2.042 & 2057. & 250.0 & 2134.3 & 25.04 & 6451 . & 0.01947 & 0.00695 \\
\hline 16. & 646.5 & 589.0 & 570.8 & 572.0 & 2086 & 1.787 & 1915. & 250.0 & 2046.4 & 23.79 & 6009 . & 0.01971 & 0.00710 \\
\hline 17. & 647.1 & 588.7 & 571.3 & 572.4 & 2087 & 1.602 & 1797 & 250.0 & 1946.9 & 22.73 & 5642. & 0.02007 & 0.00722 \\
\hline 18. & 647.5 & $58 R .0$ & $571 . ?$ & 572,3 & 2087. & 1.418 & 1679. & 250.0 & 1854.9 & 21.66 & 5274 . & 0.02040 & 0.00737 \\
\hline 19. & 648.5 & 586.9 & 570.9 & 571.9 & 2088 & 1.202 & 1516. & 250.0 & 1733.7 & 20.08 & 4767 & 0.02125 & 0.00757 \\
\hline 20. & 649.8 & 586.1 & 570.9 & 571.9 & 2088. & 1.004 & 1377. & 250.0 & 1627.9 & 18.72 & 4334. & 0.02155 & 0.00777 \\
\hline 21. & 665.9 & 605.2 & 575.2 & $577 . ?$ & 2079. & 6.216 & 3671. & 249.0 & 4143.4 & 40.26 & 11248 & 0.01800 & 0.00625 \\
\hline 22 . & 667.0 & 603.9 & 575.5 & 577.6 & $208 t$. & 4.982 & 3263. & 249.0 & 3825.0 & 36.91 & 10007 & 0.01839 & 0.00645 \\
\hline 23. & 670.0 & 600.8 & 575.2 & 577.0 & 2095. & 3.541 & 2675 . & 249.0 & 3443.2 & 32.85 & 8225 & 0.01965 & 0.00700 \\
\hline 24. & 669.4 & 600.0 & 576.0 & 577.6 & 2100 & 2.524 & 2272 & 249.0 & 2932.0 & 27.80 & 6997 . & 0.01953 & 0.00697 \\
\hline 25. & 672.2 & 597.1 & 575.7 & 577.1 & 2104. & 1.536 & 1745. & 249.0 & 2435.1 & 22.62 & 5386. & $0.0 ? 032$ & 0.00739 \\
\hline 26. & 645.6 & 590.5 & 570.6 & 472.1 & 2097. & 2.046 & 2441. & 222.0 & 2497.9 & 29.87 & 7646. & 0.01999 & 0.00699 \\
\hline$? 7$ & 845.2 & 590.3 & 570.4 & 572.0 & 2097. & $2.03 !$ & 7441. & 240.0 & 2407.1 & 29.85 & 7651. & 0.01940 & U.'נ0648 \\
\hline 28. & 845.2 & 500.6 & 571.2 & 572.4 & $\geq 006$. & 2.946 & 2444. & $? 72.0$ & 2479.8 & 29.84 & 7660 . & 0.01993 & 0.00697 \\
\hline 2\%: & 645.0 & 590.3 & 571.2 & 572.3 & 2096. & 2.948 & 2443. & 310.0 & 2482.7 & 29.84 & 7658. & 0.01998 & 0.00698 \\
\hline 30. & 645.0 & 500.1 & 571.2 & 572.2 & .2096 & 2.949 & 2452. & 376.0 & 2501.0 & 29.92 & 7699. & 0.01985 & 0.00697 \\
\hline 21. & 645.3 & 590.1 & 571.4 & 572.3 & $209 t$. & 2.950 & 2446. & 448.0 & 2507.4 & 29.85 & 7672 . & 0.01994 & 0.20697 \\
\hline 32. & 645.6 & 500.3 & 571.7 & 572.6 & 2096. & 2.949 & 2447 & 550.0 & 2513.9 & 29.84 & 7073. & 0.01992 & 0.00696 \\
\hline $33:$ & $6 ? 2.7$ & 598.7 & 570.9 & 572.3 & 2059. & 6.728 & 3714. & 251.0 & 2381.2 & 39.61 & $1175 ?$ & $0.0179 ?$ & 0.00810 \\
\hline 34. & 624.0 & 587.0 & 571.0 & 972.9 & 2067. & 5.004 & 3290. & 351.0 & 2210.6 & $3 h .37$ & 10416 & 0.01847 & 0.00632 \\
\hline 35. & 625,8 & 586.2 & 571.0 & 5.72 .1 & 2075. & 3.519 & 2604. & 251.0 & 1978.7 & 32.13 & 8540 & 0.01953 & 0.00682 \\
\hline 26. & 628.4 & 508.0 & 571.6 & 572.5 & 2077. & 1.443 & $2 \dot{0} 40^{\circ}$. & 351.17 & 1612.8 & 24.55 & 6403. & $0.0192 t$ & 0.00686 \\
\hline 37. & $6 \times 1.5$ & $58 \div .2$ & 571.2 & 572.1 & 2083. & 1.018 & 1390. & 251.0 & 1247.0 & 18.51 & 4417. & 0.02148 & 0.00762 \\
\hline 38. & 689.2 & 615.4 & 580.4 & 582.6 & 2062 & $t .118$ & $357 \mathrm{~h}$ & 307.0 & 4913.8 & 39.41 & 10760 . & 0.01821 & 0.00627 \\
\hline 30. & 689.7 & 614.1 & $=81.3$ & 583.4 & 2068 & 5.010 & $3 \geqslant 20$ & 307.0 & 4526.6 & 36.62 & 9707. & 0.01853 & 0.30647 \\
\hline 40. & 694.4 & $60^{\circ} .8$ & 570.6 & 581.5 & 2078 . & 3.523 & 2629 & 302.0 & 4139.6 & 32.42 & 7950. & 0.01979 & 0.00702 \\
\hline 41. & 697.3 & 606.2 & 577.9 & 579.5 & 2085 & 2.019 & 2009. & 302.0 & 3403.6 & 25.17 & 6091 . & 0.01968 & 0.30713 \\
\hline 12. & 600.6 & 600.9 & 578.7 & รan. & 2089. & 1.015 & 1361 . & 302.0 & 2498.5 & 18.92 & 4156 & 0.02177 & 0.00791 \\
\hline
\end{tabular}


SURFACE NUMBER 11.

\begin{tabular}{|c|c|c|c|c|c|c|c|c|c|c|c|c|c|}
\hline RUN & $\begin{array}{l}A I R \\
\text { IIN } \\
\text { OEG } R\end{array}$ & $\begin{array}{l}\text { AIR OUT } \\
\text { OEG } P\end{array}$ & $\begin{array}{l}\text { H2O IN } \\
\text { DEG R }\end{array}$ & $\underset{D E G}{H 2 O}$ OUT & $\begin{array}{l}\text { AIR MEAN } \\
\text { PRES SURE } \\
\text { PSFA }\end{array}$ & $\begin{array}{l}\text { CORE DP } \\
\text { IN. H H OO }\end{array}$ & $\begin{array}{l}\text { AIR } \\
\text { FLOW } \\
\text { SCFM }\end{array}$ & $\begin{array}{l}\text { WATER } \\
\text { FLDW } \\
\text { GPM }\end{array}$ & $\begin{array}{l}\text { HEAT } \\
\text { TRANSFER } \\
\text { RTU/MIN }\end{array}$ & $\begin{array}{l}\text { AIR HEAT } \\
\text { TRANSFER } \\
\text { CDEFF. } \\
\text { BTU/HR FT F }\end{array}$ & $\begin{array}{l}\text { AIR } \\
\text { REYNOLDS } \\
\text { NUMBER }\end{array}$ & 5 & J \\
\hline 1. & $t 36.6$ & 610.5 & 569.5 & 571.8 & 2206. & 10.320 & 3192. & 87.5 & 1546.7 & 26.02 & 14405 . & 0.01603 & 0.00298 \\
\hline 2 . & 637.1 & 609.2 & $569 . t$ & 571.7 & 2180 & 8.060 & 2783. & 79.0 & 1443.1 & 24.70 & 12571 & 0.01633 & 0.00324 \\
\hline 3. & 639.1 & 608.9 & 570.1 & 572.0 & 2165 & 6.430 & 2461. & 88.0 & 1384.5 & 22.14 & 11110 & 0.01657 & 0.00329 \\
\hline 4. & 638.3 & 608.3 & 570.1 & 572.0 & 2155. & 6.370 & 2437. & 95.5 & 1355.7 & 22.09 & 11011. & 0.01668 & 0.00331 \\
\hline 5. & 640.5 & 606.2 & 570.2 & 572.0 & 2133. & 4.000 & 1901. & 85.5 & 1209.6 & 18.94 & $8594=$ & 0.01 .712 & 0.00364 \\
\hline 6. & 641.4 & 604.7 & 570.2 & 571.9 & 2123. & 3.010 & 1623. & 84.0 & 1107.8 & 17.11 & 7347 & 0.01763 & 0.00385 \\
\hline 7. & 644.0 & 602.7 & 570.3 & 571.8 & 2113. & 1.980 & 1304. & 84.0 & 1000.9 & 15.00 & 5905. & 0.01796 & 0.00421 \\
\hline 8. & 645.0 & 599.3 & 571.0 & 572.3 & 2158. & 1.008 & 947. & 84.0 & 803.9 & 12.06 & 4300. & 0.01784 & 0.00466 \\
\hline 9. & 629.2 & 598.1 & $5 \in 0: ?$ & 562.1 & 2125. & 5.100 & 2174. & 84.0 & 1254.7 & 20.02 & 9943. & 0.01683 & 0.00337 \\
\hline 10. & 631.1 & 596.1 & $5 \in 0.5$ & 562.1 & $211 c$. & 3.460 & 1758. & 84.0 & 1142.6 & 17.85 & 8045 . & 0.01743 & 0.00372 \\
\hline 11. & $633 . t$ & 593.3 & 560.6 & 562.0 & 2095. & 2.050 & .1326 & .84 .0 & 990.8 & 14.97 & 6076. & 0.01815 & 0.00413 \\
\hline 12. & 636.2 & 589.9 & 560.8 & 561.9 & 2127. & 1.000 & 915. & 93.0 & 786.9 & 11.50 & 4204 . & 0.01894 & 0.00460 \\
\hline 13. & 6.34 .0 & 593.1 & 560.6 & 562.0 & 2109. & 1.900 & 1267. & 94.0 & 962.2 & 14.43 & 5809. & 0.01849 & 0.00417 \\
\hline 14. & 635.0 & 591.0 & $560 . ?$ & 562.1 & 2142 . & 1.200 & 1017. & 85.0 & 831.7 & 12.23 & 4670. & 0.01849 & 0.00440 \\
\hline 15. & 640.7 & 586.3 & 560.7 & 561.7 & 2140 & 0.500 & $62^{\circ}$. & 84.0 & 635.6 & 8.99 & 2895 . & 0.02028 & 0.00523 \\
\hline 16. & 628.0 & 598.8 & 559.9 & 562.0 & 2159. & 5.725 & 2334. & 74.0 & 1268.3 & 21.16 & 10674 . & 0.01664 & 0.00332 \\
\hline 17. & 628.2 & 598.2 & $560 . c$ & 561.8 & 2159. & 5.710 & 2323 & 83.0. & 1295.2 & 21.18 & 10627. & 0.01677 & 0.00334 \\
\hline 18. & 628.4 & $54 \% .8$ & 560.3 & 562.0 & 2159. & 5.685 & 2330. & 100.0 & 1325.1 & 20.96 & 10063. & 0.01660 & 0.00329 \\
\hline 19. & 629.7 & 557.0 & 560.4 & 562.0 & 2159. & 5.690 & 2320 & 119.0 & 1369.4 & 20.75 & 10007. & 0.01677 & 0.00328 \\
\hline 20. & 629.5 & 557.0 & 60.9 & 562.1 & 2159. & 5.720 & 2337 & 150.0 & 1412.0 & 21.16 & 10098. & 0.01663 & 0.00331 \\
\hline 21. & $6 \geq 0 . t$ & 596.8 & 560.9 & 562.0 & 2159. & 5.700 & 2323. & 179.0 & 1459.8 & 21.23 & 10028. & 0.01678 & 0.00335 \\
\hline 22. & 633.0 & 501.4 & 60.7 & 561.8 & $215 e$. & 1.508 & 1150. & 85.0 & 829.3 & 13.57 & 5293. & 0.01829 & 432 \\
\hline 23. & 636.0 & 589.6 & $5 n 0.7$ & 561.8 & 2154. & 0.990 & 912. & 84.0 & ?85.4 & 11.52 & 4192. & 0.01912 & 0.00463 \\
\hline 24. & 637.6 & 589. & 61.0 & & 2152. & 0.800 & 827. & 84.5 & 738.7 & 10.57 & 3798. & 0.01879 & 468 \\
\hline 25. & 639.4 & 587.2 & 560.9 & 561.9 & 2151. & 0.600 & 110. & 84.5 & 687.6 & 9.89 & 3265. & 0.01920 & 0.00511 \\
\hline 26. & 627.1 & 600.2 & 560.1 & 561.8 & 2180 & 9.780 & 3100 & 114.0 & 1550.3 & 24.69 & 14165 & 0.01620 & 0.00292 \\
\hline 27. & 628.4 & 599.0 & 560.4 & 562.1 & 2149 & 7.200 & 2628. & 114.0 & 1439.1 & 22.41 & 12412 . & 0.01641 & 0.00312 \\
\hline 28. & 640,1 & 585.5 & 561.1 & $5 \mathrm{Al} \cdot \mathrm{\theta}$ & 2!1!. & 0.500 & 620. & $11+, 0$ & บิ ติT. 2 & 8.96 & 2045. & 0.02029 & 0.00523 \\
\hline 29. & 641.8 & 584.2 & 561.3 & 562.0 & 2130. & 0.400 & 552. & 114.0 & 591.2 & 8.34 & 2546. & 0.02105 & 0.00554 \\
\hline 30. & 643.6 & 522.2 & 561.3 & 562.0 & 2124. & 0.300 & 428 & 114.0 & 488.3 & 6.87 & 1979. & 0.02613 & 0.00588 \\
\hline 31. & 647.0 & 580.0 & 561.1 & 561.7 & 2123. & 0.200 & 372. & 114.0 & 462.2 & 6.55 & 1721 & 0.02326 & 0.00646 \\
\hline 32. & 635.7 & 579.9 & 555.8 & 556.7 & 2142. & 0.400 & 582. & 30.5 & 604.4 & 8.82 & 2702. & 0.01919 & 0.00555 \\
\hline 32. & 636.2 & 579.0 & 555.7 & 556.6 & 2142 & 0.360 & 542. & 80.5 & 575.5 & 8.39 & 2516. & 0.01995 & 0.00567 \\
\hline 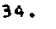 & & & 5.8 & & 41. & 0.3 & 13. & 80.5 & 59. & 8.08 & 381 . & 0.019 & \\
\hline
\end{tabular}


SURFACE NUMBER 12

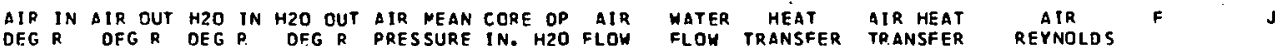
DEG R OFG R DEG P. DFG R PRESSURE IN. H2O FLOW FLOW TRANSFER TRANSFER REYNOLDS

BTU/HR FT F.

$\begin{array}{lllllllllllllll}1.616 .8 & 601.5 & 555.4 & 556.7 & 2159 . & 2.430 & 2978 & 76.0 & 843.6 & 43.96 & 38251 . & 0.04257 & 0.00743\end{array}$

2. $616.7 \cdot 600.6 \quad 555.3 \quad 556.4 \quad 2142 . \quad 2.000 \quad 2666.076 .0 \quad 797.5 \quad 41.14 \quad 34264.0 .04342 \quad 0.00777$

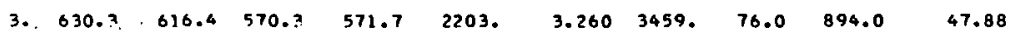

$\begin{array}{llllllllll}4 .-632.6 & 614.7 & 570.6 & 571.6 & 2131 \text {. } & 1.480 & 2241 \text {, } 76.0 \quad 746.0 & 37.69\end{array}$

$\begin{array}{lllllllllll}5 . & 633.6 & 613.1 & 570.9 & 571.0 & 2006 & 0.965 & 1771 . & 76.0 & 674.6 & 33.81\end{array}$

6. $634.4 \quad 610.0 \cdot 571.1 \quad 571.8 \quad 2081 . \quad 0.485 \quad 1162 . \quad 76.0 \quad 527.9 \quad 25.78$

$\begin{array}{lllllllllll}7 . & 636 . P & 609.2 & 571.2 & 571.9 & 2074 . & 0.270 & 858 & 76.0 & 439.7 & 20.58\end{array}$

8. $437.8 \quad 608.7564 .4 \quad 565.2 \quad 2093$. $0.395 \quad 1162.76 .0 \quad 628.6 \quad 27.00$

$\begin{array}{lllllllllll}9 . & 638.0 & 607.8 & 564.6 & 565.3 & 2090 & 0.300 & 949 . & 76.0 & 548.3 & 23.03\end{array}$

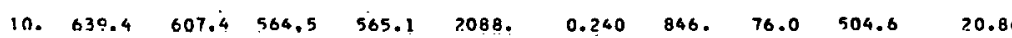

11. 4.39.2 $606.2564 .6 \quad 565.4 \quad 2087.0 .220 .821 . \quad 76.0 \quad 504.0 \quad 21.28$

$\begin{array}{lllllllllll}12 . & 431.5 & 616.1 & 563.8 & 565.2 & 2211 . & 3.400 & 3498 . & 76.0 & 999.8 & 47.20\end{array}$

13. $631.8 \quad 615.1 \quad 563.9 \quad 565.2 \quad 2173$. $2.550 \quad 3009 . \quad 76.0 \quad 034.9 \quad 43.62$

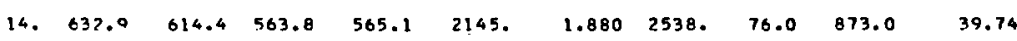

15. $634.4^{\circ} \quad 612.6564 . ? \quad 565.4 \quad 2117$. $1.085 \quad 1882 . \quad 76.0 \quad 763.4 \quad 33.96$

16. $608.7 \quad 504.0 \quad 553.3 \quad 554.4 \quad 2127$. $1.704 \quad 2456.49 .0 \quad 666.5 \quad 39.62$

17. $608.8 \quad 594.0 \quad 553.5 \quad 554.5 \quad 2128$. $1.708 \quad 2464.65 .0 \quad 674.6 \quad 38.85$

18. 608.A $543.7 \quad 553.7 \quad 534.0 \quad 2127, \quad 1.700 \quad 2455.02 .0 \quad 677.2 \quad 38.45$

10. $608.9 \quad 593.8 \quad 553.7 \quad 554.4 \quad 2127 . \quad 1.700 \quad 2461.100 .0 \quad 689.3$

20. $600.2 \quad 503.6 \quad 553.8 \quad 554.4 \quad 2128.01 .707 \quad 2461 . \quad 130.0 \quad 709.7$

$\begin{array}{llllllllll}21 . & 605.0 & 593.3 & 554.2 & 554.7 & 212 \text { A. } & 1.701 & 2474 . & 1 \text { 月O.0 } & 720.5\end{array}$

38.32

38.99

39.52

22. $635.1 \quad 611.1559 .7 \quad 560.8$

2105." 1.000 1812. 79.0 806.1

33.31

73. $636.5 \cdot 610.9559 .9 \quad 561.0$

2098.0 .795 1609. $0.0 \% 767.7$

31.16

24. 636.1609 .9560 .1561 .1

2095. 0.705 1508. 78.0 734.1

30.00

43651 . 0.042020 .00696

28273. 0.044290 .00846

22369 . 0.045640 .00960

14704. 0.053120 .01116

$10854,0.054170 .01206$

14690.0 .043940 .01169

11985 . $0.05000 \quad 0.01222$

10700 . 0.050220 .01240

10390. $0.04907 \quad 0.01304$

44172 . $0.04303 \quad 0.00878$

37975. $0.04302 \quad 0.00729$

32033 - 0.044130 .00787

23756 . 0.045980 .00508

31852 . 0.043790 .00814

31966 . 0.043590 .00795

31852 . $0.04374 \quad 0.00790$

31924 . 0.043530 .00785

31928. 0.043720 .00797

32106. 0.043150 .00205

22891 * 0.045590 .00925

20315. $0.04590 \quad 0.00974$

19056. 0.046350 .01001

?a. $636.7 \quad 609.7540 .2561 .7$

2052. 0.605 1379. $79.0+02.2$

27.67

17418 . 0.047550 .01010

26. $635.2 \quad 611.4 \quad 559.6 \quad 560.8$

211c. $\quad 1.0001821 \cdot 70.0^{\circ} 804.3$

33.42

22995. $0.04526 \quad 0.00523$

27. $635.2 \quad 611.4 \quad 559.6 \quad 560.7$

2110. 1.050 1821. $90.0 " 806.0$

33.05

23002 0.047440 .00912

28. $635.4 \quad 611.6 \quad 559.0 \quad 560.9$

2110. 1.000 1813. 90.0 909.2

32.88

$23888 \cdot 0.045640 .00912$

29. $435.4 \quad 611.4 \quad 559 . \mathrm{c} \quad 560.8$

211c. 1.0001813 .104 .0917 .0

32.98

22897. $0.04564 \quad 0.00915$

30. 835.7 611.t 560.1560 .9

$1.0001921 .120 .0 \quad 816.0$

$2,2998 . \quad 0.04524 \quad 0.00899$ 
SURFACE NUMBER 13

\begin{tabular}{|c|c|c|c|c|c|c|c|c|c|c|c|c|c|}
\hline RUIN & $\begin{array}{l}\text { AIR IN } \\
\text { DEG R }\end{array}$ & $\begin{array}{l}\text { AIR OUT } \\
\text { OFG R }\end{array}$ & $\begin{array}{l}\text { H2O IN } \\
\text { OEG }\end{array}$ & $\begin{array}{c}\text { HzO OUT } \\
\text { OEF, R }\end{array}$ & $\begin{array}{l}\text { AIR MEAN } \\
\text { PRES SURE } \\
\text { OSFA }\end{array}$ & $\begin{array}{l}\text { CORE OP } \\
\text { IN. HZO }\end{array}$ & $\begin{array}{l}\text { AIR } \\
\text { FLOH } \\
\text { SCFM }\end{array}$ & $\begin{array}{l}\text { HATER } \\
\text { FLON } \\
\text { GPM }\end{array}$ & $\begin{array}{c}\text { HEAT } \\
\text { TRANSFER } \\
\text { BTU/MIN. }\end{array}$ & $\begin{array}{l}\text { ATR HEAT } \\
\text { TRANSFER } \\
\text { COEFF. } \\
\text { BTU/HR FT F }\end{array}$ & $\begin{array}{c}\text { A IR } \\
\text { REYNOLDS } \\
\text { NUNBER. }\end{array}$ & $s$ & J \\
\hline 1. & 643.7 & 604.0. & 563.2 & 566.4 & 2139. & 15.290 & 3185. & 80.0 & 2340.7 & 75.37 & 12040 : & 0.09525 & 0.01062 \\
\hline 2. & 653.4 & 594.7 & 564.2 , & $566.0^{a}$ & 2137. & 3.575 & 1462 . & 80.0 & 1595.0 & 46.73 & 5554 : & 0.09600 & 0.01436 \\
\hline 3. & 651.9 & 595.5 & 565.3 & 567.4 & 2154. & 3.790 & 1532 & 80.0 & 1606.0 & 48.42 & 5820. & 0.09334 & 0.01419 \\
\hline 4. & 656.4 & $: 593.4$ & 565.8 & 567.7 & 2149. & 2.475 & 1208. & 80.0 & 1415.1 & 41.29 & 4592 . & 0.09814 & 0.01535 \\
\hline 5. & 658.1 & 591.2 & 565.6 & 567.5 & 2148 & 1.900 & 1039. & 80.0 & 1291.5 & 37.61 & 3958 . & 0.10206 & 0.01626 \\
\hline 6. & $6 \times 2.6$ & 588.7 & 566.0 & 567.6 & 2146. & 1.235 & 833. & 80.0 & 1144.5 & 33.04 & 3181. & 0.10342 & 0.01781 \\
\hline 7. & 653.9 & 599.4 & $565 . ?$ & 567.3 & 2161. & 3.010 & 1358. & 80.0 & 1503.6 & 44.45 & 5163. & 0.09476 & 0.01470 \\
\hline B. & 655.9 & 592.4 & 565.8 & 567.7 & 2159. & 2.220 & 1149. & 80.0 & 1357.6 & 40.13 & $43760^{\circ}$ & 0.09786 & 0.01568 \\
\hline 9. & 659.6 & 500.3 & 585.8 & 567.4 & 2158 & $1.590^{\circ}$ & $968:$ & 80.0 & 1246.5 & 36.23 & 3691 . & 0.09901 & 0.01681 \\
\hline 10 & 629.9 & 573.2 & 560.4 & 561.1 & 2124. & 0.695 & 609. & 120.0 & 640.3 & 25.69 & 2390. & 0.11170 & 0.01903 \\
\hline 11. & 628.9. & 573.4 & 560.3 & 561.0 & 2123. & 0.800 & 660. & 120.0 & 689.6 & 28.09 & 2626. & 0.10653 & 0.01893 \\
\hline 12. & 628.4 & 574.3 & 560.3 & 561.0 & 2124. & 0.995 & 754. & 120.0 & 757.6 . & 30.61 & 2958 . & 0.10405 & 0.01829 \\
\hline 13. & 626.2 & 576.6 & 560.1 & 580.9 & 2127. & 1.493 & 941. & 120.0 & $867: 3$ & 34.21 & 3681 . & 0.09998 & 0.01638 \\
\hline 14. & 622.1 & 581.0 & 559.4 & 561.0 & 2127. & 3.090 & 1384. & 71.0 & 1058.1 & 44.59 & 53 & 09492 & \\
\hline 15. & 622.4 & 520.8 & 559.5 & $561 . c$ & 2127. & 3.085 & 1384. & 80.0 & 1068.5 & 44.09 & 5394. & 0.09481 & 0.01435 \\
\hline 16. & 622.6 & 80.5 & 59.8 & 561.1 & 2127. & 160 & 1385. & 00.0 & 1084.3 & 43.48 & 5399. & 401 & 0.01414 \\
\hline 17. & 623.5 & 580.1 & $5 \in 0.1$ & 561.2 & 2126. & 3.090 & 1385. & 130.0 & 1117.0 & 43.51 & 5400. & 0.09496 & 0.01415 \\
\hline 18. & 623.4 & 19.5 & 60.4 & ib 3 & 2126. & 180 & 1385 & 160.0 & 1127.0 & 43.70 & 5406 . & 0.00467 & 0.01421 \\
\hline 19. & 631.0 & 573.7 & 559.9 & 560.7 & 2122 & C. 807 & 668. & 120.0 & 711.0 & & 2619. & 0.10749 & 0.01874 \\
\hline 20. & 631.8 & & 60.0 & 5 & 121. & & 608. & 120.0 & 665.7 & & & & \\
\hline 21. & 632.8 & 572.2 & 560.0 & 560.6 & 2120 & 0.600 & 583. & 120.0 & 655.5 . & 26.03 & 2290. & 0.10526 & 0.02013 \\
\hline 22 . & 639.6 & 89.3 & 59.9 & 561.9 & 2161. & .050 & 1601. & 80.5 & 1497.4 & 48.11 & 6145 . & 0.09274 & \\
\hline 23. & $6 ? 5.8$ & 592.3 & 560.7 & 563.0 & 2172 & 6.110 & 2015. & 80.0 & 1630.1 & 54.73 & 7722. & 0.08843 & 0.01221 \\
\hline 24. & 634.4 & 594.0 & 60.9 & 563.3 & $>182$. & .050 & 2347. & 80.0 & $17 \in 2.9$ & 60.69 & 8985. & 0.08606 & 0.01162 \\
\hline 25. & 632.9 & 596.2 & 561.0 & & & 11.450 & & 80.8 & 1858.4 & 66.20 & 10740. & 0.08600 & 0.01059 \\
\hline 26. & 632.2 & 597.0 & 560.9 & 563.6 & 2218. & 13.100 & 3030 & 80.0 & 1984.3 & 70.38 & 11581. & 0.08490 & 0.01044 \\
\hline 27. & 634.1 & 594.8 & 563.9 & & 2168. & .570 & 2533. & 120.0 & 1850.4 & & 9694 . & 0.08715 & 0.01102 \\
\hline 78. & 635.1 & 592.6 & $5 \in 5.4$ & 560.8 & 2143 & 5.650 & 1953. & 120.0 & 1578.6 & 53.48 & 7485. & 0.08601 & 0.01231 \\
\hline 27. & 637.5 & & & & $213 t$. & 4.470 & 1695. & 120.0 & 1450.6 & 48.36 & 6502. & 0.09013. & 0.01283 \\
\hline 30. & 646.7 & & & 564.4 & .2135. & $1.3 \mathrm{AO}$ & 980. & 120.0 & 1038.7 & 32.89 & 3397. & 0.10455 & 0.01681 \\
\hline 31. & 648.7 & 581.0 & 562.9 & $563 . \mathrm{e}$ & 2138 & 1.015 & 752. & 120.0 & 946.6 & 29.94 & 2910. & 0.10558 & 0.01790 \\
\hline
\end{tabular}




\section{APPENDIX B}

Literature Tube Bank Results 


\section{THIS PAGE WAS INTENTIONALLY \\ LEFT BLANK}

$z^{i}$

$\because \because 1$ 


\section{APPENDIX B}

\section{LITERATURE TUBE BANK RESULTS}

Three sets of data were taken from the open literature to help determine the effects that tube pitch had on tube bank performance. Two sets of data were for finned tube banks, and one set was for bare tube banks.

Ackerman and Brunsvold ${ }^{5}$ reported results with six arrangements of studded tubes. There were 6 studs per row on a 0.25 -in. staggered pitch, tube $O D=$ $1.875-i n$, and $A_{f} / A_{t}=0.878$. The results were transformed to $f$ and $j$ curves using tube material and free flow areas described by Worley and Ross.? Tube bank designations and basic design data equations are listed in table B-l. In all cases, the minimum free flow area was in the transverse spacing between tubes.

Jameson ${ }^{6}$ reported results with a number of arrangements of helically finned tubes. The data listed in table B-l are an interpretation of Jameson's results and figure 10-81 in reference 4. The tabulated results assumed 9.05 fins per inch, a $0.774-i n$. tube $O D$ and $A_{f} / A_{t}=0.912$. The minimum free flow area was in the transverse tube spacings for all banks except $\mathrm{J}-\mathrm{D}$, where the minimum was in the diagonal spacings.

Bare tube bank data results were taken from reference 4. Curves of basic design data plus pertinent geometric information for 8 arrangements of $0.375-i n .-O D$ bare tubes were given in reference 4: figures 10-5, 10-6, $10-7,10-8,10-9,10-10,10-13$, and 10-14. These arrangements are designated $K \amalg-5, K \amalg-6, K \amalg-7, K \amalg-8, K L-9, K L-10, K \amalg-13$, and $K L-14$, respectively. Transverse ard longitudinal spacinge for these tube banks are listed in table B-2. 
Table B-1

FINNED TUBE BANK PERFORMANCE DATA

\begin{tabular}{|c|c|c|c|c|c|c|c|c|c|c|c|}
\hline Tube Bank & $S_{t}$ & $\mathrm{~S}_{1}$ & Type* & $\mathrm{D}$ & $\alpha$ & Re Range & a & $\mathrm{m}$ & $\mathrm{b}$ & $\mathrm{n}$ & Reference \\
\hline$A B-1$ & 5.0 & 3.5 & $S$ & 0.0853 & 24.6 & $7600-27000$ & 0.2375 & -0.202 & 0.520 & -0.453 & 5 \\
\hline$A B-2$ & 6.0 & 3.25 & $S$ & 0.1094 & 22.1 & $9800-35000$ & 0.360 & -0.202 & 0.627 & -0.453 & 5 \\
\hline$A B-3$ & 5.0 & 4.5 & $S$ & 0.1097 & 19.1 & $9800-35000$ & 0.234 & -0.202 & 0.595 & -0.453 & 5 \\
\hline$A B-4$ & 5.0 & 4.5 & I & 0.1097 & 19.1 & $9800-35000$ & 0.0392 & -0.069 & 0.490 & -0.453 & 5 \\
\hline$A B-5$ & 5.0 & 3.25 & S & 0.0792 & 26.5 & $7000-25000$ & 0.240 & -0.202 & 0.513 & -0.453 & 5 \\
\hline$A B-6$ & 4.0 & 3.5 & $S$ & 0.0528 & 30.8 & $4700-17000$ & 0.128 & -0.202 & 0.391 & -0.453 & 5 \\
\hline $\mathrm{J}-\mathrm{A}$ & 1.557 & 1.75 & $S$ & 0.01681 & 138.2 & $1100-7100$ & 0.198 & -0.25 & 0.105 & -0.319 & 6 \\
\hline$J-B$ & 1.982 & 1.75 & S & 0.02689 & 35.1 & $1700-11000$ & 0.287. & -0.25 & 0.121 & -0.319 & 6 \\
\hline $\mathrm{J}-\mathrm{C}$ & 2.725 & 1.75 & $\mathrm{~S}$ & 0.04453 & 51.8 & $2800-19000$ & 0.400 & -0.25 & 0.143 & -0.319 & 6 \\
\hline$J-D^{* *}$ & 2.725 & 0.80 & S & 0.01587 & 135.3 & $1300-8500$ & 0.222 & -0.25 & 0.0866 & -0.319 & 6 \\
\hline$J-E$ & 1.982 & 1.375 & $\mathrm{~S}$ & 0.02113 & 108.2 & $1350-9000$ & 0.284 & -0.25 & 0.112 & -0.319 & 6 \\
\hline
\end{tabular}

**Minimum free-flow area in diagonal spacings. 
Table B-2

BARE TUBE BANK ARRANGEMENTS

\begin{tabular}{|c|c|c|c|c|}
\hline Tube Bank & $S_{t}$ & $\mathrm{SI}_{1}$ & Type* & Reference \\
\hline $\mathrm{KL}-5$ & 0.5625 & 0.4688 & S & 4 \\
\hline $\mathrm{KL}-6$ & 0.4688 & 0.4688 & $\mathrm{~S}$ & 4 \\
\hline $\mathrm{KL}-7$ & 0.5625 & 0.375 & $S$ & 4 \\
\hline$K L-8$ & 0.5625 & 0.5625 & $S$ & 4 \\
\hline $\mathrm{KL}-9 * *$ & 0.750 & 0.375 & $S$ & 4 \\
\hline$K L-10^{* *}$ & 0.9375 & 0.2812 & $\mathrm{~S}$ & 4 \\
\hline$K L-13$ & 0.563 & 0.469 & $I$ & 4 \\
\hline$K L-14$ & 0.469 & 0.469 & I & 4 \\
\hline \multicolumn{5}{|c|}{$\begin{array}{l}\text { *S = staggered, } I=\text { in-line. } \\
\text { *Minimum free-flow area in diagonal spacings. }\end{array}$} \\
\hline
\end{tabular}




\section{ACKNOWLEDGMENTS}

The author gratefully àcknowledges the contributions of R. R. Uglow and Dr. G. J. Kidd to this study. 


\section{REFERENCES}

1. Kern, D. Q. and Kraus, A. D., Extended Surface Heat Transfer, McGrawHill, New York, 1972.

2. Cox, B. and Jallouk, P. A., Experimental Determination of Performance Characteristics of Eight Compact Heat Transfer Surfaces, Union Carbide Corporation, Nuclear Division, Oak Ridge Gaseous Diffusion Plant, Oak Ridge, Tennessee, December 27, 1972 (K-1832). UNCLASSIFIED.

3. Cox, B. and Jallouk, P. A., Methods for Evaluating the Performances of Compact Heat Transfer Surfaces, ASME Paper 72-WA/HT-56, 1972.

4. Kays, W. M. and London, A. L., Compact Heat Exchangers, 2nd Ed., McGraw-Hill, New York, 1964.

5. Ackerman, J. W. and Brunsvold, A. R., "Heat Transfer and Draft Loss Performances of Extended Surface Tube Banks," Journal of Heat Transfer, Trans. ASME, Series C, Vol. 92, May 1970, pp. 215-220.

6. Jameson, S. L., "Tube Spacing in Finner Tube Banks," Trans. ASME, Vol: 67,1945 , pp. 633-639.

7. Worley, N. G. and Ross, W., "Heat Transfer and Pressure Loss Characteristics of Cross-Flow Tubular Arrangement with Studded Surfaces" (Paper 2), The Use of Secondary Surfaces for Heat Transfer with Clean Gases, Institution of Mechanical Engineers, Great Britain, November 1960, pp. 15-26.

8. Neal, S. B. H. C. and Hitchcock, J. A., A Study of the Heat Transfer Processes in Banks of Finned Tubes in Cross Flow, Using a Large Scale Model Technique, Proceedings of the Third International Heat Transfer Conference, August 7-12, 1966, pp. 290-298.

9. Kays, W. M., London, A. L., and Lo, R. K., "Heat Transfer and Friction Characteristics for Gas Flow Normal to Tube Banks - Use of a Transient Test Technique," Trans. ASME, April 1954, pp. 387-396.

10. Boucher, D. F. and Lapple, C. E., "Pressure Drop Across Tube Banks Critical Comparison of Available Data and of Proposed Methods of Correlation," Chemical Engineering Progress, Vol. 44, No. 2, February 1948, pp. 117-134. 
THIS PAGE

WAS INTENTIONALLY

LEFT BLANK 
DISTRIBUTION

INTERNAL

1-2. Administrative Offices
Stief, S. S.
Winkel, R: A.

3- 5. Capacity Expansion Program Dalton, J. T. Huber, A. P. Jones, J. K.

6. Computing Technology Center

7-11. Engineering Division Crump, B. F., Jr. Kellogg, D. R. Papke, D. Patton, F. S. Senatore, S. J.

12-13. Fabrication \& Maint. Div. Krieg, E. H., Jr. studinger, L. A.

14-28. Gaseous Diffusion Dev. Div. Ayers, W. D: Brady, J. P. Brock, W. R. Burton, D. W. Collins, W. T. Fulmer, U. C. Higgins, R. L. Jallouk, P. A. Kidd, G. J. King, C. J. McGill, R. M. Pashley, T, H. Trammell, H. E. Uglow, R, R. Vance, J. M.

29. Laboratory Division Napolitan, D. S.

30-38. Lihrn.ry

Fraser, R. J.

39-42. Operations Analysis \& LFP Div. 75-79. Ebel, R. A. Lang, D. M. Noritake, H. M. Pasquier, L. P.

EXTERNAL

80-248: TID-4500
43-45. Operations Division Cates, P. S. Legeay, A. J . Patterson, G. P.

47. Osborne, H. H.

47. Sommerfeld, K. W.

48-50. Separation Systems Cox, B. Evans, E. C. Szady, A. J.

51. Technical Director Wilcox, W. J., Jr.

52-54. ORGDP. Records Department (RC)

55-56. U.S. Atomic Energy Commission Keller, C. $\Lambda$. Zachry, D. S.

57-64. Goodyear Atomic Corporation Brown, R. W.

Earnhardt, M. M.

Langebrake, C. 0 . Netzer, W. D.

Tabor, C. B. Woltz, F. E. Zangr $\mathrm{L}, \mathrm{B}$. J. Zeek, R. M.

65-66. ORNL Fee, G. G. Hof fman, H. W.

67-74. Paducah Plant Bernstein, $S$. Clifford, W. R. Golliher, W. R. Hopkins, C. C. Levin, R. W. Loveland, C. W. Merriman, J. R. Riepe, R. C.

Y-12 Flant

Case, J. M. Griffin, J. D. Horde, G. W. Jasny, G. R. Jordan, R. G. 